\title{
Un contrepoint contesté : appropriation « juive » et libération polyphonique
}

Contested Counterpoint: "Jewish" Appropriation and Polyphonic Liberation

\section{Karen Painter}

Traducteur : Fanny Gribenski et Julien Ségol

\section{OpenEdition \\ Journals}

Édition électronique

URL : http://journals.openedition.org/transposition/321

DOI : 10.4000/transposition.321

ISSN : 2110-6134

Éditeur

CRAL - Centre de recherche sur les arts et le langage

\section{Référence électronique}

Karen Painter, « Un contrepoint contesté : appropriation « juive » et libération polyphonique »,

Transposition [En ligne], 2 | 2012, mis en ligne le 01 mai 2012, consulté le 14 novembre 2019. URL :

http://journals.openedition.org/transposition/321 ; DOI : 10.4000/transposition.321

Ce document a été généré automatiquement le 14 novembre 2019.

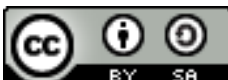

La revue Transposition est mise à disposition selon les termes de la Licence Creative Commons Attribution - Partage dans les Mêmes Conditions 4.0 International. 


\title{
Un contrepoint contesté : appropriation « juive » et libération polyphonique
}

\author{
Contested Counterpoint: "Jewish" Appropriation and Polyphonic Liberation
}

\section{Karen Painter}

Traduction : Fanny Gribenski et Julien Ségol

\section{NOTE DE L'ÉDITEUR}

Initialement publié sous le titre « Contested Counterpoint: “Jewish" Appropriation and Polyphonic Liberation ", in Archiv für Musikwissenschaft, 58e année, H 3, 2001, p. 201-230.

\section{NOTE DE L'AUTEUR}

Une version de cet article a été lue à l'Université Libre de Berlin. Je remercie Joseph Auner, Leon Botstein, Reinhold Brinkmann, Bryan Gilliam, Charles S. Maier, Albrecht Riethmüller, et Alex Ross pour leurs commentaires. J'ai bénéficié de l'aide de project. Therese Muxeneder du Centre Arnold Schoenberg (Vienne) ; de Krista Traunsteiner au Département de la Musique de la Bibliothèque Nationale d'Autriche (Vienne) ; et du personnel du Geheimes Staatsarchiv (Berlin).

1 Il est conventionnellement admis que l'émancipation de la dissonance conduisit à l'atonalité, ce qui sépara irrémédiablement le compositeur de son public, devenu hostile. Si ce raisonnement est discutable sur un plan technique, il est trompeur sur le plan historique. Le contrepoint nouveau eut d'importantes conséquences sur l'écoute musicale et, à une époque décisive, devint la métaphore même de l'art moderne. L'enjeu était bien plus important que dans d'autres aspects de l'innovation musicale la dissonance non réglée ou la sensualité de couleurs timbriques. La dissonance avait 
déjà mis à l'épreuve le public bien avant 1900 et - fait fascinant quoique méconnu de l'histoire de la réception-, les développements du contrepoint ont bien davantage suscité l'ire que ceux du langage harmonique. Le contrepoint représentait pourtant la quintessence de l'identité musicale allemande et constituait le point de départ historique de la musique occidentale. Sa réappropriation par des compositeurs dont le dessein, aux yeux de la critique, menaçait la tradition musicale, n'en était que plus dérangeante et subversive - quand bien même leur musique eût rencontré le succès auprès du public.

2 La tension entre tradition et modernité envahit l'écriture contrapuntique de bon nombre de compositeurs après 1900. Les cas les plus emblématiques sont ceux de Reger et de Busoni, mais leur musique ne provoqua pas de réactions aussi fortes et hostiles que celles de Mahler et de Schoenberg. Le contrepoint de Reger n'a pas un profil aussi marqué. Chez lui, les voix ne sont pas aussi fortement indépendantes que chez Mahler et Schoenberg. Reger semble utiliser l'harmonie pour assouplir la structure, faisant du contrepoint un élément de stabilisation ${ }^{1}$. La musique pour piano (comme la Fantasia contrappuntistica) ainsi que les opéras de Busoni, n'étaient pas aussi strictement associés à un genre que la symphonie ou le quatuor à cordes pour Mahler et Schoenberg. À l'aube du nouveau siècle, alors que la critique commençait à se donner pour objectif la protection de la tradition, le contrepoint de Mahler et Schoenberg fut souvent l'objet d'attaques antisémites. Or, il s'agissait là de bien davantage que d'un énième réquisitoire de la critique culturelle antisémite. Le thème de l'antisémitisme s'était en effet complexifié depuis la rédaction du texte classique de l'antisémitisme musical, « Das Judentum in der Musik» (Wagner, 1850). Il n'était pas non plus assimilable à l'antisémitisme politique allemand, austro-hongrois ou français. Certains commentateurs juifs de Vienne se faisaient même l'écho de cet antisémitisme pour se différencier des juifs de l'Est qu'ils considéraient comme incultes.

3 Les réactions au contrepoint doivent également être comprises dans le contexte de ce que nous suggérons être une orientation différente des critiques viennois et allemands, tant sur le plan culturel que national. Ces enjeux apparaissent clairement à la lecture des comptes rendus d'une série de créations données au cours de la saison 1906-1907 (voir Annexe 2, ci-dessous). La création viennoise de la Sixième Symphonie de Mahler eut lieu deux semaines avant celle du Quatuor à cordes opus 7 de Schoenberg : la critique du contrepoint des deux œuvres se fonda sur le stéréotype du juif assimilé. Les termes du débat changèrent lors de la création allemande du quatuor de Schoenberg, donné au festival de Dresde avec Salomé de Strauss. Le "Quintette du Juif», dans Salomé, déclencha un antisémitisme plus virulent encore à l'encontre de Schoenberg, sur fond d'allusions au stéréotype du juif de l'Est non-assimilé. Pour rendre pleinement compte de ces faits, il convient de revenir sur la façon dont la trajectoire personnelle du compositeur fut perçue en Allemagne. En 1907, il était encore possible pour les critiques allemands de manifester leur mépris envers Schoenberg, qu'ils présentaient comme un juif non-assimilé. Il avait vécu pendant près de trois ans à Berlin, où il avait été directeur musical d'un cabaret. Son insuccès l'avait ramené à Vienne, où il n'avait aucune garantie d'emploi. Mahler, quant à lui, avait dirigé l'opéra Impérial de Vienne jusqu'à l'été 1907. Mais ce sont les jugements esthétiques sur leurs compositions qui nous intéressent ici.

4 Comment expliquer de si vives réactions à l'écoute du contrepoint? D'une part, le contrepoint était alors la technique musicale la plus traditionnelle et la plus rigoureuse. 
En 1901, à un moment critique de son développement, Mahler évoquait l'influence de Bach en termes presque serviles, alors qu'il commençait à s'intéresser davantage aux formes traditionnelles et au contrepoint dense. Cette tendance se manifeste dans sa Cinquième Symphonie, instrumentale et ostensiblement non programmatique, de même que dans les Sixième et Septième Symphonies qui la suivirent immédiatement. «Je n'ai pas de mots pour dire à quel point j'apprends de Bach, constamment et de plus en plus

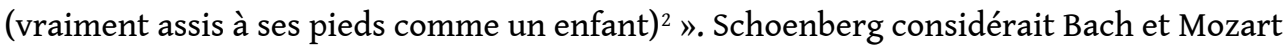
comme ses principaux maîtres et, du premier, il apprit surtout le contrepoint ${ }^{3}$. Par ailleurs, plusieurs anecdotes renvoient au contrepoint comme procédé relevant de l'intime. Chaque soir, à l'heure du coucher, le jeune Mozart chantait en contrepoint avec son père et l'embrassait sur le nez avant de s'endormir. Sur le bateau qui l'emmenait de New York en Europe, Busoni rendait quotidiennement visite à Mahler dans sa cabine, muni d'une bouteille de vin, et distrayait le compositeur, allongé sur son lit de mort, par l'interprétation « de contrepoints comiques et insensés ${ }^{4}$ ».

La spécificité du contrepoint (et de la polyphonie) en fit le relais privilégié de fonctions symboliques et idéologiques. En effet, son écoute donne l'impression d'être dans la musique, de percevoir les relations internes à sa structure, et d'être immergé dans ses différentes voix. Alors que les critiques furent fréquemment hostiles aux innovations percevant l'invention thématique comme froide ou le rythme comme insaisissable et tourmenté - le contrepoint leur interdit toute distance vis-à-vis du matériau compositionnel. L'état de vulnérabilité qu'il implique a fait du contrepoint l'objet d'interprétations politiques. Il fit naître une sensation de malaise pouvant aller jusqu'à l'anxiété quand il fut ressenti en tant que contrepoint. Les discours esthétiques sur le contrepoint, nous le verrons, soulignent l'idée de contrôle et de coordination entre les voix, alors que le mot « polyphonie » renvoie à l'idée de leur indépendance et put, de ce fait, incarner un sentiment de liberté. (Le Troisième Reich introduisit cependant une importante exception sémantique, puisque le mot Kontrapunkt, peut-être trop académique ou technique, fut remplacé par les termes Mehrstimmigkeit et Polyphonie dans l'usage populaire et critique.)

\section{La polyphonie moderne}

6 Les aspirations mélodiques viennoises et la suspicion à l'égard de la polyphonie ont peut-être poussé Mahler et Schoenberg à considérer ce procédé comme un terrain d'innovation. Alors que le contrepoint se caractérise par une forte relation rythmique entre les voix, la polyphonie implique une grande disparité. Mahler prônait l'indépendance maximale des différentes voix, aussi bien pour l'orchestration que pour l'écriture polyphonique en général. " Dans la véritable polyphonie », explique Mahler, et à la différence du Ring de Wagner, « les thèmes se développent côte à côte et de façon indépendante, chacun de sa source à son terme propre, et se différencient autant que possible les uns des autres. " Le principe le plus important dans la composition, explique-t-il ailleurs, réside dans «la pureté des lignes - c'est-à-dire dans le fait que chaque voix [...] se doit d'être lyrique. » Même dans «la structure orchestrale la plus complexe, on doit tendre à la plus grande pureté de la voix principale ${ }^{5}$ ».

Mahler comprenait l'indépendance des voix en termes spatiaux. A la façon d'Ives faisant l'expérience d'écouter deux orchestres situés aux coins opposés d'une ville et jouant dans des mètres et des tonalités différents, Mahler se souvint d'une scène de son 
enfance, au cours de laquelle, se trouvant à l'extérieur, il percevait des sons parvenant de multiples sources, et proposa la définition suivante de la polyphonie: "c'est précisément de cette façon, depuis des directions complètement différentes, que les thèmes doivent émerger. Ils doivent en outre se caractériser par ce même degré de différence dans leur rythme et dans leur mélodie. $»^{6}$ En règle générale, les voix contrapuntiques de Mahler ne servent pas à définir de thème - on n'identifie pas de sujet mélodique, mais plutôt une interaction de voix dans l'espace, une sorte d'absence de sujet. L'orchestration accentue souvent l'indépendance des voix, comme dans les solos de violon et de trompette du premier mouvement de la Troisième Symphonie (aux chiffres [39]-[40]). La différenciation rythmique peut aussi jouer un rôle important. Dans les mesures qui suivent les deux coups de marteau du finale de la Sixième Symphonie, le rappel du thème prend la forme de lignes douloureusement augmentées aux cuivres, qui ne parviennent pas pour autant à prendre le contrôle du passage. L'étourdissante figuration des cordes se déploie de façon autonome. L'auditeur est obligé d'écouter entre les voix et se trouve dans l'impossibilité de les penser en tant que développement musical unifié. On pourrait dire que l'une des voix doit sembler démesurée, se différencier $d u$ "flou » général - pour utiliser la métaphore de la perspective visuelle.

8 Schoenberg a souvent fait preuve d'un virulent conservatisme à l'égard du contrepoint. La violence de ses objections à la soi-disant conception linéaire du contrepoint, devenue si populaire au début du vingtième siècle, est difficile à comprendre. Cependant, son désir de maintenir un contact de note contre note entre les lignes contrapuntiques est cohérent avec la conception esthétique qui sous-tend son écriture contrapuntique - bien plus que chez Mahler. L'opus 7 est remarquable à cet égard. Malgré le profil résolument rythmique des différentes voix, chacune est structurée de la même façon. C'est par exemple le cas du passage qui suit la lettre [A], caractérisé par une forte interdépendance entre les voix. Schoenberg indique même aux musiciens comment s'élabore cette structure : au moyen de croches à [A 22] et de syncopes à [A 31]. Ce passage s'inscrit dans une remarquable intensification du contrepoint imitatif qui s'étend sur cinquante-quatre mesures [A 1-54]. Après la fugue initiale [A 1-20], Schoenberg accroît la tension par une succession de variations de tempo [A 21, 31 et 43]. À chaque changement, le violoncelle s'arrête de sorte que son retour abrupt une ou deux mesures plus tard semble donner une autre dimension au développement musical. $\mathrm{Au}$ premier changement de tempo ("nach und nach etwas lebhafter ») le thème de transition du violon est en augmentation, en blanches égales (comme à [A1]), ce qui met en valeur l'intensité du contrepoint en croches. Au second changement («immer belebend»), l'élan procède d'une ligne contrapuntique plus directe de demi-tons, portant l'indication "drängend» (au second violon à [A 31-33] et au premier à [A 34-36]), qui se prolonge jusqu'à la fin de la section. Au dernier changement de tempo (« wieder ziemlich bewegt; steigernd und beschleunigend»), le thème de transition est dynamisé par une écriture en croches qui insuffle une impulsion rythmique uniforme à cette dernière section.

9 La différenciation rythmique des voix contrapuntiques, quoique répondant à l'idéal de Mahler, rendit le contrepoint de l'opus 7 de Schoenberg difficile à écouter pour ses contemporains. Les motifs superposés ne se rejoignent pas, et pourtant, l'impérieuse impression d'unité qui se dégage du matériau implique que l'auditeur perçoive les temporalités autonomes de deux ou plusieurs voix. La seconde reprise, à la fin de la section scherzo, qui constitue l'articulation principale entre les deux moitiés du Quatuor, 
constitue un bon exemple de ce phénomène. Le thème principal, au contour bien dessiné et doublé à l'alto et au second violon, se déploie contre deux lignes contrapuntiques aux profils inconciliables, écrites respectivement en triolets et en croches. Les trois lignes indépendantes continuent fortissimo pendant dix mesures jusqu'à atteindre le point culminant du registre à [I 50], après quoi la tension diminue jusqu'à la fin du mouvement (ou de la section, vu l'effort de Schoenberg pour remettre en cause le découpage traditionnel des mouvements), dans une descente progressive vers le grave et une dilution de la complexité contrapuntique.

La complexité rythmique n'est en général pas considérée comme constitutive des innovations ou provocations du modernisme musical viennois. Néanmoins, les problèmes posés par sa perception dépassèrent de loin ceux que générait la dissonance. La différenciation rythmique qu'implique le contrepoint exige un fractionnement de l'attention de l'auditeur si celui-ci veut suivre plus d'une voix à la fois. Les critiques, et particulièrement ceux qui assistèrent à la création viennoise de l'opus 7 de Schoenberg, trouvèrent cet aspect dérangeant. Albert Kauders fut gêné par le thème de l'ouverture, "quasi insipide ", et par «sa rythmique urbaine " (un thème antisémite fréquent) entraînant la perte de son caractère mélodique. Il critiqua particulièrement l'absence de repos et de répétition. (Voir l'annexe 1, ci-dessous, pour les citations de comptes rendus.) Walter Reizler jugea grotesque «l'exagération du style du quatuor" par Schoenberg, et "pire encore, que le rythme ne puisse en aucun cas être saisi par l'oreille, du fait de la mise en valeur rythmique de différentes voix ». L'écriture harmonique de Schoenberg s'inscrit dans la continuité des pratiques du dix-neuvième siècle et, si dangereuse qu'ait pu paraître la dissolution de la tonalité, la stratification rythmique était plus difficile à appréhender.

\section{L'idéal allemand du contrepoint}

Pour les Allemands, le contrepoint représentait une dimension essentielle de la musique, et d'autant plus après 1900, lorsque les règles de l'harmonie et les conventions de l'instrumentation furent confrontées à d'innombrables exceptions et innovations. Il n'est pas surprenant qu'un manuel de contrepoint comme celui de Felix Draeseke avance qu'un «développement sain de la musique» se doit d'inclure le contrepoint ${ }^{7}$. La Musikästhetik de Karl Grunsky s'inscrit dans la même perspective : c'est à travers le « conflit contrapuntique " que « le compositeur s'approche de l'essence de la musique $»^{8}$.

12 Le contrepoint était alors considéré comme un moyen de structurer et de contrôler la musique. Il offrait la promesse d'une différenciation dans un monde mal à l'aise face à la subjectivité et aux insaisissables contours de l'art fin-de-siècle, tel que le caractérisa en 1892 le très populaire ouvrage du critique Max Nordau, Entartung :

Le thème [...] n'a de cesse de perdre de sa clarté, [...] il se dissout dans un brouillard au sein duquel l'imagination est libre de percevoir la forme qu'elle désire [...]. La marée des sons afflue et reflue en d'incessantes montées et descentes chromatiques de triolets, sans limites ni but identifiables'.

13 L'angoisse de la perte de contrôle inspira tout une série de métaphores. Draeseke déplorait qu'un "gris indéfinissable" surgît du mélange de toutes les couleurs en peinture et qu'il en fût de même quand les timbres et les harmonies se confondaient. Il estimait que la musique contemporaine était le reflet des problèmes de son époque, 
ceux d'une société en flux permanent - « condamnée à un mouvement (Verkehr) sans répit $^{10} »$. Grunsky eut recours à de plus humbles métaphores, qui pourraient figurer dans un texte d'introduction à l'esthétique.

Un compositeur qui s'efforce de ne pas mélanger ses voix dans une bouillie de sons prend toujours un plaisir naturel à accroître leur énergie dans la lutte et le conflit.

Chaque voix est comme "porteuse d'une volonté ", chacune aspire au même objectif, d'où l'apparition de tensions, comme cela arrive avec la volonté humaine. En cela, la tension contrapuntique doit être comprise comme une lutte. La "supériorité d'esprit " contraint les voix à se rassembler en une "lutte pour l'unité ». Pour Grunsky, toute tentative d'augmentation de la tension qui ne s'accompagne pas d'un effort pour structurer l'intensification de la polyphonie aboutit à un effet d'affaiblissement. La tension perd de sa force et le compositeur ne parvient à rien, si ce n'est à un « bruyant jacassement de voix sans fin ». La maîtrise provient de l'interruption de la tension des segments mélodiques de lignes individuelles. C'est par ce procédé que le compositeur peut atteindre des objectifs précis ${ }^{11}$.

Les défenseurs de Mahler du nord de l'Allemagne, si peu nombreux fussent-ils (hormis à Hambourg où son travail de chef d'orchestre entre 1891 et 1897 lui avait acquis de loyaux alliés), admirèrent son contrepoint pour toutes ces qualités. Max Loewengard, critique hambourgeois et auteur d'un traité de contrepoint, qualifia le contrepoint de la Cinquième Symphonie d'« authentique » car, indépendamment du nombre de voix ou de leur brutalité (rücksichtslos), "chacune d'entre elle obéit à une légitimité logique et tend, dans une clarté directionnelle, vers un seul et même but ». Friedrich Brandes, qui devint plus tard l'éditeur de la Neue Zeitschrift für Musik, respectait la Sixième Symphonie de Mahler pour son mépris d'une "soi-disant polyphonie, notoire, parfaitement médiocre et familière ». Les termes de l'éloge étaient cependant ambigus. Selon Brandes, aucun compositeur de son temps ne possédait une maitrise aussi froide du contrepoint (littéralement, de " combinatoire ») que Mahler. Brandes s'appuyait sur le stéréotype du juif assimilé comme intellectuel dépourvu d'émotion : " le savoir-faire de Mahler ne lui apporte aucune inspiration, mais des idées et des combinaisons ». Brandes ne fit jamais référence à la force expressive de la musique. Il était plutôt impressionné par « les connaissances littéraires » de Mahler, au point de conclure que "son sérieux, sa finesse, sa confiance imposent le plus profond respect, au même titre que sa maîtrise. »

16 Les attentes en matière de traitement contrapuntique dépendaient en grande partie du genre musical. Dans la symphonie, le contrepoint se devait de donner une impression de force allant jusqu'à submerger l'auditoire - en accord avec la tradition du sublime. Dans ce contexte, Bruckner, l'archétype du contrapuntiste, servait de référence. Selon le critique et professeur berlinois Adolf Weißmann, le compositeur s'appuyait principalement sur le procédé traditionnel de l'inversion et évitait les "nuances" contrapuntiques typiques de la musique moderne. Pour lui, la monumentalité primitive de la symphonie brucknérienne (" toutes ses symphonies ne font qu'une ») ne peut être pleinement ressentie que «dans l'humilité la plus absolue ${ }^{12} »$. Dans son ouvrage dédié au compositeur, Rudolf Louis estime que Bruckner aspire à " exprimer l'impossible » à travers « la combinaison contrapuntique » des principaux thèmes dans le finale :

On ne peut qu'être saisi et ébahi par sa capacité à mouvoir de gigantesques blocs thématiques ; ceux-ci façonnent des édifices raffinés et solides qui surpassent les facultés humaines aussi bien que divines. Il empile les uns sur les autres ces colosses inamovibles avec une force incroyable ; s'ensuit une lutte à mort avec cette matière 
incontrôlée. C'est un drame d'un sublime rare[, dont on ne peut que difficilement appréhender tout] le pouvoir et la grandeur (sic) ${ }^{13}$. avait une fonction politique ou sociale) ne concernait pas le genre du quatuor à cordes. L'humilité prônée par Weißmann pour l'écoute de Bruckner aurait été inadéquate dans le contexte intime de la musique de chambre. En outre, les harmonies audacieuses avaient leur place en des lieux plus vastes, tels les salles de concert ou les opéras, mais pas dans la musique de chambre. Le compte rendu de l'opus 7 de Schoenberg paru dans la Neue Musik-Zeitung de Berlin expliqua que, du point de vue de la critique musicale, toute cacophonie était exposée dans un quatuor à cordes, "servie sur un plateau "; seule la force des grands orchestres avait la faculté "d'améliorer, d'adoucir et d'élever " la matière sonore de cette façon. Le critique ajouta que l'opus 7 avait divisé l'auditoire tout comme Salomé : sa brillante orchestration avait détourné le public peu averti de la perception de la cacophonie, alors que le musicien et «le critique professionnel » déplorèrent ce qu'ils avaient entendu et vu sur la partition. On peut imaginer que la disposition des musiciens ait renforcé le contraste des attentes vis-à-vis des musiques symphonique et de chambre. Le positionnement des quatre musiciens sur la scène inscrit la musique dans un rapport d'immédiateté, alors que, face à une masse orchestrale, le regard du public est concentré sur le chef. En général, l'opus 7 pose le même défi que le contrepoint symphonique (rapports thématiques organiques, travail thématique, dynamiques contrastantes), en même temps qu'il résiste ingénieusement à la cohésion symphonique, envisagée sous l'aspect rythmique et spatial. Il n'est donc pas surprenant que le moment contrapuntique crucial de la symphonie, le rappel thématique en vue de la conclusion, n'ait pas fait l'objet de mentions dans les premières critiques du Quatuor.

L'écriture contrapuntique força l'admiration des Allemands, presque indépendamment de sa complexité ou de ses conséquences harmoniques. Cela valut même pour les critiques aux orientations esthétiques et politiques opposées, tels que Max Marschalk, du journal libéral Vossische Zeitung, et Rudolf Louis, brucknérien et antisémite notoire : Schoenberg est "certainement le contrapuntiste le plus audacieux et téméraire de notre temps » s'exclama Marschalk. Louis, quant à lui, estima que Schoenberg dirigeait "les quatre voix du quatuor à cordes d'une manière extrêmement contrapuntique, c'est-à-dire avec une absolue indépendance ». Les deux critiques s'abstinrent de tout jugement définitif sur le Quatuor, invoquant la nécessité de le réentendre et d'étudier la partition. "Sa musique n'est pas de celle dont j'ai besoin comme nourriture quotidienne de mon âme", note Marschalk, alors que Louis se demande si le contrepoint de Schoenberg « jaillit d'une impulsion créatrice véritable et naturelle » ou 
«nait d'une réflexion purement rationnelle » - rhétorique familière de l'antisémitisme. Mais finalement, la fascination exercée par le contrepoint amena Louis à laisser de côté ses réticences pour faire l'éloge du compositeur juif. Pour lui, l'opus 7 était « peut-être [le travail] le plus intéressant» du festival et son écoute le stimula à tel point qu'il conclut: "j'examinerai certainement plus d'une fois la partition de ce quatuor inhabituel pour rafraîchir, développer et clarifier les impressions que son exécution fit sur moi ». De fait, il manifesta beaucoup moins d'enthousiasme à propos du Trio pour piano en fa mineur de Wilhelm Rohde, également au programme. Il le décrivit comme " une élégante petite musique de chambre, séduisante, mais de peu d'intérêt. »

Même lorsqu'il fut l'objet de critiques, le contrepoint inspira tout de même le respect. Pour les critiques impartiaux, la " polyphonie sans règles » qui assiège les tympans de l'auditeur, comme l'écrivait Adolf Weißmann suite à la création berlinoise de l'opus 7 , entraîna le développement audacieux d'une esthétique du «made at home " (en anglais dans le texte). Weißmann expliqua que cela correspondait précisément au vœu de Schoenberg: ce qu'un critique considérait comme « du talent et du baume pour les oreilles", Schoenberg le condamnait comme une faiblesse. La capacité de ce compositeur à atteindre ses objectifs lui valut le respect de Weißmann et d'autres critiques allemands. "Rien n'est plus instructif " que de comparer Schoenberg aux compositeurs de la « Nouvelle École française » programmée au festival. «La musique de Schoenberg est une musique barbare, mais écrite avec l'énergie vitale d'un autodidacte fanatique ", alors qu' "un Français comme Gustave Samazeuilh [...] crée sans énergie vitale, d'une manière totalement froide, en suivant une recette déterminée. "

\section{Le contrepoint à Vienne}

Les critiques viennois ont tous montré beaucoup moins d'attirance pour le contrepoint que leurs homologues allemands. Malgré l'auguste tradition d'enseignement du contrepoint au conservatoire de Vienne (Bruckner, Simon Sechter et Robert Fuchs), les Viennois n'écrivirent pas de traités de contrepoint, du moins pas jusqu'à la publication de Kontrapunkt de Schenker en 1910 et de Grundlagen des linearen Kontrapunkts. Bachs melodische Polyphonie de Kurth en 1917 (Schoenberg eut l'intention d'écrire un traité du contrepoint, mais les demandes qu'il fit à ses éditeurs ne reçurent pas de réponses, et ses ébauches esquissées en 1911 n'aboutirent jamais ${ }^{14}$ ). À Vienne, au début du vingtième siècle, le Gradus ad Parnassum de Fux faisait toujours office de référence pour le contrepoint ${ }^{15}$.

Cette division culturelle et géographique entre Vienne et l'Allemagne se retrouve dans les métaphores utilisées pour désigner le contrepoint. Considérons le finale virtuose de la Cinquième Symphonie de Mahler. Pour Loewengard, originaire du nord de l'Allemagne, « la puissante fugue et la strette exaltante » faisait du finale la clef de voûte de toute la symphonie. À l'inverse, Gustav Altmann, originaire du sud du pays, reconnut au finale son "contrepoint généreux", mais trouva le résultat artificiel: "Strauss est un meilleur peintre («Maler») », alors que Mahler n'a offert qu'un simple «bouquet (" Strau $\beta »)$ de fleurs artificielles». Le critique viennois Theodor Helm, qui ne perçut qu'une "musique burlesque et grotesque", signala avec dédain que, pour un enthousiaste berlinois, ce finale évoquait l'architecture de la cathédrale de Cologne. Helm (bien qu'étant lui-même un élève du contrapuntiste Bruckner) affirma que le 
scherzo n'était pas la "merveille contrapuntique " annoncée lors de la création à Cologne. Selon lui, on aurait plutôt dû s'étonner de "la témérité insensée de cette excentrique polyphonie " réalisée à partir d'un thème aussi grossier. Les commentateurs comme Helm fondèrent leurs critiques sur le fait que l'invention mélodique de Mahler ne méritait ni un tel traitement polyphonique, ni une instrumentation aussi ample, comme si le contrepoint n'avait en lui-même aucune valeur.

Cette ambivalence viennoise envers le contrepoint transcende les clivages. Les critiques musicaux progressistes aussi bien que les défenseurs de la tradition intensifièrent leurs attaques à la suite des créations de 1906-1907. Prenons le cas du plus fervent partisan viennois de Mahler, Julius Korngold, et celui de son détracteur, Robert Hirschfeld. Hirschfeld dénonça la nature du procédé employé par Mahler dans sa Première Symphonie et s'insurgea contre « ce honteux contrepoint où l'unique possibilité consiste à positionner les voix les unes par-dessus les autres, au lieu de les mettre les unes contre les autres ». Selon Hirschfeld, le recours à un tel contrepoint avait conduit Mahler à composer une parodie de symphonie. À partir de la Cinquième Symphonie de Mahler, les critiques viennois dénoncèrent tant l'abondance que la nature de l'écriture contrapuntique. Dans le cas de Korngold, on peut considérer sa critique initiale de l'écriture contrapuntique de Mahler comme neutre. Pour lui, le finale était le mouvement le plus «significatif » de la Cinquième Symphonie, non pour son contrepoint magistral, mais, précisément, parce que le contrepoint ouvrait la voie à une structure mélodique élargie: "Il y règne un flux qui englobe toute l'amplitude et l'intensité contrapuntiques ». Mais la rhétorique du critique changea lors de la saison suivante. L'angoisse face à une culture de l'excès, si caractéristique de l'atmosphère fin-de-siècle, s'imposait. Korngold perçut alors dans le finale de la sixième symphonie une « hypertrophie du contrepoint - ou quel que soit son nom dans les termes actuels ». Ce critique libéral se dédit immédiatement de ses positions antimodernistes et recentra le problème sur la seule question de la compréhensibilité :

chaque voix, en reprenant des morceaux de thèmes, aspire à lutter pour sa propre vie. Dans ce combat, dans ce bellum contrapunticum omnium contra omnes, on ne trouve finalement que des perdants.

24 La critique de Salomé par Hirschfeld se conclut par un appel plus ciblé contre l'excès : «l'orchestre straussien, par sa polyphonie sans limite et ses infinies transitions au sein $\mathrm{du}$ spectre des couleurs instrumentales et de l'analyse harmonique, qui n'est pas sans rappeler celle des expériences de chimie, atteint dans chaque nouvelle œuvre le sommet de ce qu'il est possible de faire aujourd'hui. » À partir de ce sommet, chaque nouveau pas nous élève davantage, « l'air musical se fait plus rare ; toute la végétation de l'art musical disparaît ", et il ne reste plus de l'art que de nouvelles techniques d'escalade.

Le contrepoint est finalement devenu la bête noire (sic) de la musique moderne. En 1909, dans son compte rendu de la création viennoise de la Septième de Mahler, Korngold s'unit aux voix d'autres critiques pour attaquer « les modernes de la musique » comme Schoenberg et Dukas, qui faisaient preuve d'une « imagination immodérée » dans leur "marche sans répit vers de nouvelles idées harmoniques et contrapuntiques " et n'utilisaient que très peu l'harmonie dans sa véritable fonction: celle consistant «à faire fructifier les idées mélodiques.»Cet avocat de Mahler pointa néanmoins l'indépendance des voix contrapuntiques comme l'une des faiblesses du compositeur et s'aligna sur les idéaux politiques du Forum libéral dans lequel ses critiques paraissaient. 
L'« individualisation et la démocratisation sans limite des voix, tout comme leur totale juxtaposition et surimposition" attirent et repoussent tout à la fois les auditeurs, à l'instar de l'excès dans d'autres aspects de la composition ${ }^{16}$.

L'ordre particulier de ces créations a dû augmenter l'angoisse ressentie face aux développements du contrepoint. Le public fut mis à rude épreuve par la polyphonie radicale du Quatuor à cordes (comme Schoenberg devait le souligner lui-même plus tard), et il le fut encore davantage en entendant ce même contrepoint nouveau deux jours après, à quinze instruments solistes, lors de la création de la symphonie de chambre op. 9. Les critiques qui firent le compte rendu de ces deux oeuvres à la fois virent le danger latent des innovations contrapuntiques du quatuor. Pour Kauders, la Symphonie de chambre érigeait en système le principe de l'« écœurement» dans la musique. L'expérience fut apparemment si déconcertante pour Kauders qu'il n'eut de cesse de répéter quelles étaient les conséquences d'une telle écriture contrapuntique: la nouvelle œuvre n'était pas une «symphonie » mais une " antiphonie » et la diction n'y était pas régie par un principe d'harmonie mais de disharmonie. Il y avait du vrai dans cette critique de l'excès - du moins Schoenberg le pensa-t-il plus tard. Songeant aux premières exécutions, il fit remarquer que la principale difficulté de l'œuvre résidait dans « la grande quantité de contrepoint ${ }^{17}$ ». Il n'était pas seulement question du défi lancé au public. Schoenberg nota sur une réduction pour piano à quatre mains de la Symphonie de chambre : « Tout cela est beaucoup trop chargé !!! Il devrait y avoir moitié moins de voix ! ». Plusieurs années après la création, il supprima des voix entières pour alléger la texture, ce qui eut pour résultat de faire émerger plus distinctement les relations polyphoniques ${ }^{18}$. Pour Schoenberg, le contrepoint représentait un terrain d'expérimentation comportant ses propres risques d'excès. Plus le contrepoint est dense, moins les voix individuelles sont dessinées. On pourrait faire la même remarque au sujet de l'orchestration. Les révisions successives de son contrepoint par Schoenberg (pour l'alléger et le clarifier) font en effet songer à celles que Mahler apporte à son orchestration, souvent même après la création de l'oeuvre. On peut aussi rapprocher la réflexion de Schoenberg et de Mahler sur ces paramètres en ce qu'ils invoquent tous deux les notions de rigueur et de tradition. Schoenberg avait le sentiment de suivre les principes du contrepoint de Bach et Mahler voulait une orchestration dans laquelle chaque voix fût à elle-même sa propre mélodie.

Il est clair qu'il existait toujours une conception mélodique de la musique à Vienne au début $\mathrm{du} \mathrm{XX}^{\mathrm{e}}$ siècle. Les compositeurs ne se sentaient pas nécessairement contraints par une telle conception - en fait, la mélodie devint une métaphore pour expliquer leurs innovations. Songeons notamment aux métaphores utilisées par Schoenberg pour désigner sa théorie des douze sons et à l'affirmation de Mahler selon laquelle l'orchestration consiste à écrire une mélodie pour chaque voix de l'orchestre. Les critiques musicaux utilisèrent aussi le concept de mélodie comme une sorte de métaphore de l'écoute musicale. Alors que les Allemandes percevaient l'intensification symphonique à travers le contrepoint ou le travail thématique, la décrivant souvent en termes héroïques, les Viennois étaient attentifs à la mélodie. Cela renvoie peut-être à la capacité de retenir un thème par cœur - aspiration ancienne dans la culture musicale bourgeoise. Même le critique du Journal des travailleurs viennois, David Josef Bach, fit observer que l'andante de la Sixième Symphonie de Mahler manquait «d'une touche capable de remuer intérieurement l'auditoire, afin que celui-ci puisse la ramener chez lui comme une nouvelle et appréciable acquisition ». Dans la tradition esthétique, une telle expérience impliquait l'acquisition d'une «vision d'ensemble» pour un 
mouvement ou une œuvre, qui formât une image durant au-delà du moment musical. Un critique musical viennois expliqua qu'avec toute oeuvre d'art, le public voulait avoir une "impression totale ", " une image claire et idéale dans toute son indétermination, qui nous frappe comme une possession amoureuse». Dans le cas de l'op. 7 de Schoenberg, continua le critique, cette "vision d'ensemble» disparut lorsque les thèmes commencèrent à tourbillonner les uns dans les autres ${ }^{19}$. Les Viennois n'étaient pas sensibles qu'à la beauté mélodique, mais aussi à la beauté du timbre, c'est du moins l'impression qu'en eut un berlinois comme Weißmann. Après la création berlinoise du quatuor, Weißmann expliqua qu'un critique impartial eût reconnu des moments de beauté et une joie toute viennoise dans la sonorité du Quatuor de Schoenberg mais que «l'insouciante Vienne avait, de façon compréhensible, rejeté cet homme ».

Le contrepoint interféra cependant avec l'écoute de la mélodie. Son effet subversif fut tout d'abord dépeint en termes humoristiques. Maximilian Muntz se plaignit du fait que, dans le scherzo de la Cinquième Symphonie de Mahler,

des thèmes de valse et de Ländler, dépouillés de leur naïve innocence et effrontément maquillés des couleurs de l'orchestre moderne, virevoltaient dans un cancan contrapuntique.

Les allusions moqueuses à la féminité étaient chose commune chez les critiques viennois, comme l'on pouvait s'y attendre de la part d'une culture journalistique qui donna naissance à la revue Die Fackel de Kraus. Mais deux ans plus tard, à la suite de la création du Quatuor de Schoenberg, les critiques évitèrent soigneusement toute référence au contrepoint, sous-entendant que la texture de Schoenberg ne relevait pas de ce noble procédé : de grossières métaphores suffirent alors pour le caractériser. Les passages de la passion lyrique à l'énergie contrapuntique furent peut-être déconcertants, comme s'ils portaient atteinte à l'humanité de la mélodie. Le journal viennois Montags Revue estima que Schoenberg "se mettait tout à coup à respirer profondément " au beau milieu d'un «combat au couteau ». Même le critique de l'un des organes politiques les plus progressistes de la ville, le Wiener Allgemeine Zeitung, vit dans les premières mesures de l'œuvre un assaut de thèmes (ce même passage devint le centre de l'essai de Berg intitulé «Pourquoi la musique de Schoenberg est-elle si difficile à comprendre ?») :

Le thème chaste est immédiatement ligoté et violé (vergewaltigt) par les autres voix ; pour l'essentiel, l'autre thème sombre les assiste dans cette tâche.

Lorsqu'une "seconde idée indépendante » fit son entrée, le critique essaya de la suivre à nouveau jusqu'à ce qu'elle fût vaincue, quoique d'une façon différente :

celle-ci semblait aussi, de son côté, complètement dépassée, écrasée, broyée et rendue folle ${ }^{20}$.

Les voix contrapuntiques indépendantes eurent donc pour effet d'envahir l'auditeur ou de le submerger.

Une conception mélodique de la musique implique la présence d'une solide ligne de basse contre laquelle le thème se développe et s'appuie. Selon Max Kalbeck, l'absence d'une telle solide fondation conjuguée à une écriture mélodique extravagante donnèrent à la Sixième Symphonie de Mahler un « caractère beaucoup trop personnel, intime, exclusif et singulier ». Hormis dans l'andante « les basses touchent à peine le sol avant de prendre une impulsion et de décoller ». Une musique dépourvue de solides fondations semblait manquer de l'objectivité et de la force requises dans les genres publics. La zone de confort entre le sujet à l'écoute et l'objet musical était anéantie. Les 
voix indépendantes du contrepoint de Schoenberg n'offraient que peu de sécurité ou d'assise. Le violoncelle n'apporte pas de ligne de basse stable (sauf à quelques endroits du scherzo), pas même lors de l'exposition du thème ou dans les passages conclusifs. La ligne active de cet instrument gêna particulièrement Kauders, à qui elle sembla « caquetante et procéder par soubresauts à la basson (sic)».

La programmation constitue un autre facteur d'explication des différences de cultures musicales et de modes de réception. La création du Premier Quatuor à cordes de Schoenberg à Vienne fut programmée avec la «musique divine» du quintette en Do majeur de Schubert - pour reprendre les termes de plusieurs critiques. Le programme de Berlin qui associa Schoenberg au dernier Beethoven connut un plus grand succès. Les critiques comparèrent les deux compositeurs et rappelèrent aux lecteurs le caractère un temps problématique de la réception de Beethoven. Cette différence dans la programmation s'inscrivait dans une tendance générale des Allemands à considérer l'exécution musicale comme un processus d'édification plutôt que comme un divertissement. Le critique du Berliner Börsen Courier eut ainsi le sentiment que les circonstances de l'exécution au festival de Dresde ne favorisèrent guère la contemplation ni le discours rationnel. La chaleur extrême ( 25 degrés Celsius à l'ombre en fin d'après-midi), les vêtements inhabituellement festifs portés par les femmes, qui remplissaient une salle comble, tels sont les détails de son compte rendu. Malgré cela, le critique se réjouit de rapporter que «le public [avait] réagi aux différentes compositions présentées lors du concert avec sérieux et enthousiasme ». Lorsque l'op. 7 fut finalement exécuté à Berlin, Weißmann songea au festival de Dresde et suggéra que "l'atmosphère de Berlin [était] plus favorable à une contemplation critique et apaisée ».

\section{«Conversation juive »}

Envisagés avec du recul, les termes du débat nous sont familiers: une admiration allemande pour un contrepoint puissant et une répulsion viennoise pour l'indépendance et la division. Il est tentant de penser aux élans nationalistes respectifs de ces deux États : les ambitions d'expansion allemandes et le souci austro-hongrois de maintenir son contrôle sur l'empire multinational. Dans ce contexte, il est surprenant que la perception d'une appropriation juive du contrepoint ait davantage troublé les Allemands que les Autrichiens. La rhétorique utilisée véhicula des significations dépassant les questions de politique musicale de la saison. Elle offre un aperçu des préoccupations face à la soi-disant opposition des identités juives et allemandes, avant que ces thèmes n'aient fait leur apparition dans la presse. Pendant ce bref moment, les stéréotypes associés aux juifs se situaient entre l'expression d'une réaction authentique (même si, à nos yeux, ce qualificatif est porteur de dangereux préjugés) contre la musique moderne et leur utilisation comme exutoire à un racisme antisémite plus général.

Dans les débats des années 1906-1907, les tropes antisémites fonctionnaient encore indépendamment de leur caractère abject - comme un véhicule du jugement esthétique. Bien sûr, une question se pose: de telles catégories auraient-elles pu émerger dans la critique musicale sans l'antisémitisme latent de la culture politique d'Europe centrale ? Quoi qu'il en soit, il est important de mettre en évidence la logique 
et la structure du discours critique antisémite, même si l'on rejette ses implications politiques et culturelles.

La critique musicale démontre à quel point l'assimilation culturelle demeurait fragile et combien il était facile pour le langage critique (même sous la plume de commentateurs juifs) d'insister sur la distinction entre une identité allemande prétendument englobante et un ensemble de caractéristiques soi-disant juives qui refusaient de se mêler au creuset national. De plus, les stéréotypes juifs étaient d'une souplesse dangereuse ; ils pouvaient être utilisés pour décrire la musique en termes élogieux, neutres ou négatifs ou servir à rejeter une composition dans son ensemble. Ils pouvaient également être invoqués, même par les critiques, sans aucune raison esthétique, en se basant uniquement sur une sorte d'antisémitisme pur.

Les différents courants d'antisémitisme peuvent être particulièrement bien étudiés à travers les métaphores associées au discours, qui offrent un lieu commun récurrent dans les discussions sur le contrepoint. Ces métaphores abondent dans la critique du contrepoint imitatif. L'abbé Vogler avait comparé l'indépendance des voix dans la fugue à une foule agitée ${ }^{21}$. Schoenberg lui-même méprisait l'imitation approximative dans la polyphonie moderne, qu'il considérait comme un faible substitut au véritable contrepoint. Il vilipenda ce qu'il appelait le «contrepoint Rhabarber», du mot Rhabarber, utilisé au théâtre lorsque des gens imitent le son d'une foule en émeute derrière la scène $\mathrm{e}^{22}$.

Au début du vingtième siècle, les métaphores associées à la communication avaient une visée pratique. L'expression des émotions ne constituait plus la fonction première de la nouvelle musique et les critères esthétiques de modération, de clarté et de symétrie semblaient aussi passés de mode. Le succès d'une œuvre dépendait au contraire de la capacité du compositeur à transmettre ses idées au public. Dans ce contexte, la noble métaphore de la musique comme langage fut ramenée au rang de "conversation ", puis de simple "bavardage ». Il y avait là bien davantage qu'une intensification de la critique rhétorique, habituelle dans le domaine de la presse musicale. Il s'agissait aussi d'un changement dans la fonction des thèmes.

Les métaphores associées au discours pouvaient désigner un art dénué de sens spirituel - et, plus spécifiquement, le caractère éphémère d'une musique moderne fondée sur la simple performance, et non pas un art éternel exprimant la profondeur des émotions humaines. Ce type de critique s'inspirait du texte " Das Judentum in der Musik », dans lequel Wagner élabora des métaphores autour du thème « du juif cultivé, [qui] se devait de parler ", quand bien même l'expression artistique était contraire à sa nature. Une telle personne est aussi peu capable de véritables sentiments qu'un perroquet " débitant des mots et phrases humaines ${ }^{23}$. » Dans sa critique de la création de la Sixième Symphonie de Mahler à Essen, Gustav Altmann, compara le compositeur à un interlocuteur "nauséabond " qui parle avec "grande éloquence et talent technique " mais qui, soir après soir (ou d'une symphonie à l'autre ), se fait plus insistant, suffisant et bruyant dans sa "récitation de banalités ». À Vienne, où la nature discursive du langage faisait l'objet de la fascination des universitaires et des critiques, la métaphore discours/musique avait un pouvoir d'explication particulier. La métaphore de la musique comme discours n'avait pas sa place dans le genre de la symphonie et ne s'avéra d'aucune utilité pour la réception des œuvres de Mahler. Un correspondant viennois présent lors de la création berlinoise de la Sixième Symphonie proposa un jeu de mots élaboré à partir des termes «reden » et «Rhetorik » pour mettre en évidence deux 
aspects de la composition: Mahler était moins intéressé par le contenu (la matière thématique) que par la rhétorique et la présentation (une brillante orchestration), et se contentait de "parler » (de façon tantôt spirituelle, tantôt pensive ou triviale) plutôt que de "chanter». Finalement, comme pour se moquer des excès oratoires de la musique, le critique fila la métaphore jusqu'à son terme : « Imaginez un intervenant qui ne développe aucun thème, et veut seulement prononcer un discours " (Allgemeine Musik-Zeitung).

Salomé suscita des métaphores associées au discours juif à un moment où la plupart des critiques ne se sentaient pas à l'aise pour discuter de l'identité juive. Dans le « célèbre Quintette des juifs ", comme le désigna Hirschfeld après la création viennoise, Strauss met la polyphonie au service d'un débat théologique non dénué d'intérêt, mais, du fait du recours à la dissonance libre et d'une orchestration inhabituelle, sa musique prend un tour étrange. Les quatre ténors entrent un par un, suivis par la basse, puis chacun entre à nouveau dans une succession rapide. Le quintette des juifs ne constituait en aucun cas le premier exemple d'un contrepoint humoristique - on en trouvait déjà de célèbres exemples dans Falstaff et les Meistersinger. Ce n'était pas non plus le premier cas de caricature du contrepoint. Strauss lui-même avait parodié l'académisme dans Ainsi parlait Zarathoustra. Mais ce quintette poussait les usages musicaux à l'extrême. Salomé fut présentée à l'exposition nazie "d'art dégénéré », pour reprendre les termes de Strauss, du fait que les juifs y chantaient « dans la plus pure atonalité » ${ }^{24}$. Alors que Strauss a souvent recours à des ténors pour représenter des personnages ordinaires, comiques ou antipathiques, la combinaison de quatre ténors et d'une basse peut, comme le suggère Sander Gilman, faire allusion au timbre aigu et à la féminité attribués aux juifs ${ }^{25}$. La querelle des juifs heurte même l'agressif Hérode - curieux passage qui rompt l'illusion opératique du chant comme représentation de la conversation. Un tel contrepoint ne relève plus de la musique mais d'une subversion de la tradition occidentale, à travers un orientalisme que les critiques assimilés cherchaient à bannir. Korngold, un juif pleinement assimilé, en parla avec une certaine ironie. Sa critique de Salomé prit la forme d'une conversation tournant en dérision les réactions de ses collègues. Un commentateur plus conservateur relève que "Strauss est dans son élément » lorsqu'il fait le portrait « spirituel » des juifs en plein débat et que l'écriture vocale de l'opéra s'apparente à la langue yiddish. Hirschfeld, moins certain de son assimilation à la société viennoise, fut troublé par le caractère direct et explicite de l'opéra de Strauss, qui contrastait avec l'esprit et les sentiments aimables de la pièce d'Oscar Wilde sur laquelle il était basé26. Il en voulait pour preuve la scène des juifs et son « contrepoint artificiel », dans lequel seule la dynamique des voix et des gestes était perceptible. Pour Hirschfeld, la scène se trouvait ainsi vidée de tout contenu musical. En outre, la manière incessante avec laquelle l'orchestre discutait et développait chaque mot prononcé, en produisant un "langage gestuel agité et grotesque», rappelait les « juifs volubiles » de l'œuvre de Wilde.

41 Lors du festival de Dresde, la métaphore familière du quatuor à cordes considéré comme conversation prit une tournure antisémite. La vision goethéenne du quatuor comme discussion entre "quatre personnes rationnelles", comme un discours dans lequel "chacun a l'impression d'y gagner quelque chose", n'avait désormais plus cours $^{27}$. Pour Friedrich Brandes, "les largeurs de vue hypermodernes et le verbiage [étaient] des plus insupportables dans la musique de chambre ». Dans le même compte rendu, Brandes déplora le fait que le festival fût dominé par Salomé, une «œuvre sensationnaliste » et «terriblement caractéristique de la décadence». Selon lui, la 
nouvelle direction prise par la musique en général trouvait son équivalent dans la notion d'esthétisation du péché proposée par Wilde, ou, pour le dire avec Brandes, la conviction que "la dépendance convulsive est un matériau dramatique de premier ordre ». Le journaliste ne fit aucune allusion à l'écriture contrapuntique dans son commentaire de la composition elle-même, néanmoins il évoqua son «jeu polyphonique " au sujet de l'exécution habile du Quatuor Rosé. Le choc produit par la découverte de Salomé fit disparaître tout l'intérêt que Brandes avait conçu l'été précédent pour l'écriture contrapuntique de la Sixième de Mahler. L'interaction des voix pendant les cinquante minutes de l'opus 7 de Schoenberg lui parut tout simplement ennuyeuse.

Les critiques considéraient rarement l'interaction des voix comme du contrepoint. Ils la caractérisaient plutôt comme une dispute cacophonique, un autre thème antisémite popularisé par "Das Judentum in der Musik» de Wagner. Le Berliner Börsen-Courier rapporta que le quatuor de Schoenberg était

si saturé de développements thématiques incessants et antithétiques [...] que les quatre instruments se livrent une bataille des plus désagréables: sous la forme d'une totale cacophonie dans la première moitié; sous celle d'un abandon névrosé dans la deuxième.

43 Le critique du Dresdner Nachrichten se référa ironiquement au quatuor comme à un « travail» (entre guillemets), et substitua au titre original de Schoenberg (« Quatuor en un mouvement ») celui de " jérémiade cacophonique en un souffle». Il ajouta que cette œuvre ne pouvait être convenablement commentée que par un psychiatre ayant un intérêt médical pour Schoenberg et sa prétendue musique.

Quelle fut la portée de la rhétorique développée à la réception de Salomé et du Quatuor à cordes de Schoenberg ? Bien que les métaphores associées au discours fussent déjà bien connues depuis Wagner, on peut se demander si les plaisanteries prolifiques $-\mathrm{y}$ compris celles que firent les critiques juifs assimilés - à l'égard du Quintette des juifs, n'ont pas influencé l'histoire antisémite de la musique allemande de Rudolf Louis (1909). Louis dénigrait le juif culturellement allemand (qui aspire à participer à la vie culturelle allemande) et prit simplement ses distances avec les Allemands de confession hébraïque (qui produisent un art exotique) : «Si la musique de Mahler parlait juif, elle me serait peut-être simplement incompréhensible». S'inspirant de Wagner, Louis affirma que si la musique de Mahler était « horriblement dégoûtante » c'est parce qu'elle " parlait l'allemand musical, si je puis dire, mais avec un accent, un ton, et surtout avec les gestes de l'Est $\aleph^{28}$. Le plus grand crime de Mendelssohn, écrivit Wagner, fut d'imiter Bach et la tradition musicale luthérienne. Plus la musique de Mendelssohn sonnait " allemand", pire elle était. Il osait se faire passer pour un Allemand de souche alors qu'il n'était rien de plus qu'un juif. Ce sont précisément les affirmations de Wagner et leur réutilisation par Louis qui montrèrent le dangereux potentiel de la pensée musicale à glisser de la critique culturelle vers un antisémitisme plus général, traversant des frontières qui ont - peut-être de façon erronée - semblé très claires dans le domaine de la littérature. Sigurd Paul Scheichl distingue par exemple le «langage anti-juif» (comme celui de Karl Kraus et d'Otto Weininger), entendu comme une rhétorique contre le modernisme, de "l'antisémitisme», qui appelle à des actions politiques ou sociales contre les juifs ${ }^{29}$. Les critiques musicales, publiées dans les rubriques importantes de journaux à grand tirage, ont-elles contribué à la légitimation de ce courant antisémite en faisant de lui une idéologie dominante? Peut-être ne reflétaient-elles pas simplement l'antisémitisme de la Vienne de Karl Lueger - où 
l'assimilation était toujours un objectif culturel et politique -, mais ont-elles anticipé, comme un mauvais présage, l'antisémitisme précédant l'Anschluss dans lequel les juifs assimilés n'auraient plus leur place.

\section{L'ethnicité du contrepoint}

La Sixième Symphonie de Mahler représentait pour la critique un problème particulier dans la mesure où elle utilise un langage moderne à l'intérieur d'une forme traditionnelle. D'aucuns jugèrent l'œuvre presque incompréhensible, au-delà de la taille considérable de l'orchestre : la Symphonie défiait à plusieurs égards les principes esthétiques et les conventions symphoniques. L'écriture contrapuntique en était rigoureuse et le contenu thématique très unifié. La présence de quatre mouvements, dans le respect de la forme symphonique traditionnelle (trois en la mineur, et observant tous, à l'exception du finale, des formes clairement identifiables - jusque dans la reprise de l'exposition et la réexposition fidèle du premier mouvement) poussa certains critiques à parler d'une forme " classique ». Mahler avait atteint l'essence de la tradition symphonique, mais sans aucun esprit mélodique, c'est du moins ce que déploraient les critiques viennois. On trouve dans les comptes rendus de nombreuses allusions au stéréotype du juif viennois assimilé : le compositeur, un intellectuel au sang froid, s'appropriait les formes tant révérées par l'esprit allemand. Le partisan le plus influent de ce stéréotype était Otto Weininger, dont l'ouvrage Sexe et caractère avait reçut l'admiration de personnalités aussi variées que Wittgenstein, Kokoschka et Karl Kraus d'une part, Hans Pfitzner et Hitler (qui le découvrit par son mentor Dietrich Eckhart), d'autre part ${ }^{30}$. Les termes de la réflexion de Weininger s'insinuèrent dans le vocabulaire émergeant de la critique culturelle et du journalisme aussi bien du côté neutre que du côté antisémite.

Mahler dut notamment essuyer le reproche d'une création démesurément prolixe, que la critique n'avait pourtant jamais formulé à l'encontre de Mozart ou Haydn. Il y avait peut-être dans l'air le souvenir du mot célèbre de Karl Lueger, alors maire de Vienne : «mes Viennois sont toujours disposés à prendre quelque repos, il n'y a que les juifs pour préférer rester affairés ${ }^{31}$. » Max Vansca commence ainsi son compte rendu de la Sixième Symphonie :

Les symphonies de Mahler sont écrites de sang-froid. Elles font montre de clairvoyance, d'esprit et d'un habile raffinement. [...] D'où leur aspect académique, signe du plein effort d'un métier épuisant. À chaque saison sa nouvelle symphonie.

Hans Liebstöckl, quant à lui, raillait Mahler d'achever une nouvelle symphonie avant même que la précédente ait pu voir le jour au concert et associait l'aspect mécanique de la Sixième à la ville industrielle où elle fut créée, Essen : « Krupp fait des canons, Mahler se contente de symphonies. » La Sixième pêche par ses thèmes :

pour chacun on a fabriqué un visage derrière lequel se cache une machine. Ils ne sont pas animés de l'intérieur et s'écrouleraient sitôt isolés de leur contexte orchestral.

Lors de la création de son premier quatuor à Vienne, quatre semaines plus tard, Schoenberg dut subir le reproche d'une appropriation exsangue et mécanique des formes et des techniques traditionnelles - d'une musique extraordinaire par son unité thématique, sa cohésion formelle et sa profondeur, mais sans mélodie facilement identifiable. C'est surtout son écriture contrapuntique qui troublait les commentateurs. 
Pour la Montags Revue, la « composition de Schoenberg ressemble au débroussaillage de sous-bois épais; après quoi il recouvre le terrain d'un filet aux mailles remarquablement serrées. » Les critiques admettaient l'unité de structure du Quatuor, mais en dénigraient le modèle organique, trop loin du noble idéal d'une musique instrumentale transcendante. Le filet en question était « une polyphonie fastidieuse et suffocante, sans laquelle toute la composition tomberait en morceaux. » Et enfin : « on a besoin de plus que nos deux oreilles pour étudier cette nouveauté. » L'impact de ces masses sonores sur l'auditeur fut commenté le jour suivant dans Das Vaterland: chaque mesure, dans la partition de l'opus 7, contient «une intéressante combinaison contrapuntique à découvrir. » Cependant, entendre « cet enchevêtrement complexe et ces contorsions surprenantes » ne compense pas le fait que « les quatre voix n'ont rien à dire ». De l'ouverture à la conclusion du Quatuor, l'auditeur est enseveli «sous de mortes masses de sons, dans la plus austère absence de sonorité ». Le brio technique et académique est dépourvu de tout « contenu intérieur et vivant ».

Le fait de parvenir ou non à entendre le contrepoint renvoie à une relation particulière à la sonorité, qui diffère de celle des autres procédés et paramètres musicaux. Les critiques se sentirent étouffés ou oppressés par un contrepoint qu'ils pouvaient lire mais n'entendaient pas. August Püringer reprochait à Schoenberg l'arrogance dont il faisait montre en ne se souciant pas de "l'accessibilité" de sa polyphonie aux auditeurs. Les conséquences en étaient plus lourdes que dans le cas d'un rythme trop complexe ou d'une mélodie insaisissable, qui sont toujours assimilés par la conscience de l'auditeur ou, au pire, passent simplement inaperçus. S'agissant du contrepoint dense, le compte rendu de Püringer permet de se faire une idée sur les causes de la virulence des réactions à l'égard de la polyphonie moderne. On suit une mélodie et on se l'approprie, on perçoit le rythme et on le ressent même parfois : si la mélodie est clairement présentée et si le rythme n'est pas « tumultueux » ou insaisissable, alors l'un comme l'autre peuvent être perçus par la conscience de l'auditoire ${ }^{32}$. En un sens, le contrepoint s'apparente davantage à l'harmonie. L'auditoire s'approprie moins ces procédés ou matériaux qu'il ne s'immisce en eux, pour y trouver prise. Faire entendre un contrepoint inaudible, c'était oppresser l'auditeur par une masse de sons impénétrable, là où la mélodie ou le rythme seraient simplement inaperçus. Face au contrepoint, aucune distance protectrice n'était possible.

Avec une portée bien plus large en terme de public, Salomé introduisit la possibilité de séparer le soi-disant modernisme juif de l'identité ethnique, particulièrement pour les intellectuels juifs. Á en croire une anecdote passée à la postérité, Arthur Schnitzler aurait dit que le compositeur de Salomé était juif, alors que Mahler représentait le parfait compositeur allemand ${ }^{33}$. On mentionnait fréquemment Salomé dans les débats sur le modernisme et sur l'avenir de la musique, ce qui n'a d'ailleurs rien d'étonnant, étant donnée la rupture radicale que représente cet opéra par rapport aux œuvres antérieures de Strauss. Ces références étaient également communes aux critiques berlinois qui avaient fait le voyage pour assister à la création du Quatuor de Schoenberg à Vienne, ce qui les portait à comparer la nouvelle scène musicale de cette ville à celle de Berlin (Strauss était associé à Vienne, malgré ses liens avec Munich). Max Marschalk, défenseur de Mahler et critique à la Vossische Zeitung de Berlin, n'était pas au clair sur sa propre réaction à l'œuvre de Schoenberg et se rappelait à lui-même (ainsi qu'à ses lecteurs) qu'« après la musique de Salomé, il était impossible de se laisser surprendre par quelque extravagance technique.» Était-il également possible que le portrait 
audacieux des juifs brossé par Strauss ait poussé Püringer à aborder le sujet dans sa critique du Quatuor à cordes de Schoenberg? "Y a-t-il nom plus doux, plus blond, aux yeux plus bleus que celui-ci: Arnold Schoenberg?», interroge-t-il au début de son article. "Comment pourrait-on penser que, derrière ce nom emprunt d'idéalisme sentimental et vacancier, se cache un anarchisme musical de l'espèce la plus redoutable?» L'auteur clôt son propos par une discussion de la «modernité viennoise », qui avait une longueur d'avance sur «la nouveauté » berlinoise. Strauss reste « le Dionysos de notre époque, dégénéré en Bacchus par trop de convoitise des plaisirs corrupteurs. » Il « mène la tendance moderne, 'après comme avant Schoenberg', et ce par un élan (sic) séducteur.»

51 La critique du quintette des juifs fut étendue à l'opéra dans son entier. L'élite des critiques musicaux de la ville protesta contre ce qui leur semblait une exploitation des principes wagnériens : l'exacerbation des procédés de communication, qui tendait à représenter musicalement chacune des actions. L'excès des figuralismes et du recours à l'expressivité orchestrale facilitaient le rapport de l'auditeur profane à l'œuvre, alors que les critiques dénonçaient le manque de clarté de ce langage moderne dans le contrepoint et l'harmonie. De la même façon, les critiques décrivirent le contrepoint de Schoenberg en termes antisémites lors du festival de Dresde, sous l'influence, me semble-t-il, de la réception de l'opéra de Strauss. Salomé fut donné le premier soir du festival et, l'après-midi suivant, on jouait précisément le Quatuor de Schoenberg. Puisque de nombreux critiques avaient déjà commenté l'opéra lors de sa création, et qu'ils étaient toujours réticents à faire deux fois le compte rendu d'une même œuvre, ils firent du Quatuor la nouvelle cible de leurs préoccupations en termes de modernisme musical. En outre, il est probable que l'annulation à la dernière minute de la présence de Strauss, relevée par la plupart des critiques, ait d'autant plus entamé la considération pour un opéra qui passait dès lors plus pour un pilier acquis au répertoire que pour l'œuvre récente d'un compositeur mise à l'épreuve devant ses collègues $^{34}$. Les critiques rapportèrent également qu'en dépit de leur rejet presque unanime de Salomé lors de sa création, l'importante acclamation du public avait porté l'oeuvre sur les scènes allemandes. Le succès sans précédent de Salomé, lit-on dans la Neue Musik-Zeitung, faisait l'effet du Donner's Hammerblow écartant les nuages, opposant irrémédiablement la critique et le public. Les critiques étaient fâchés d'un tel succès auprès du public, mais ils avaient trouvé une nouvelle cible dans le cas de ce contrepoint moderniste bien moins susceptible d'emporter les suffrages du public. Friedrich Brandes concéda que Salomé avait suscité d'intéressants débats en 1905, mais il manifesta sa colère lorsque l'œuvre fut annoncée au programme du Festival deux ans plus tard. L'hostilité exprimée des deux côtés envers le Quatuor de Schoenberg permit de résorber l'épineux écart entre public et critiques. Quelques-uns allèrent même jusqu'à suggérer que l'opus 7 ne méritait pas une d'être joué en public. Le chroniqueur du Dresdner Nachrichten rapporta non sans une certaine fierté que le Quatuor Petro, à Dresde, avait refusé de jouer l'opus 7 de Schoenberg, alléguant « qu'il n'avait pas sa place dans une salle de concert. "

52 Les critiques les plus fermement opposés à l'opéra de Strauss eurent également recours au thème antisémite de la décadence de l'Est pour dénigrer Schoenberg. F. A. Geissler jugeait absurde qu'un auditoire de compositeurs pût être envoûté par le « charme » de Salomé. De même, il reprocha au Quatuor Rosé d'avoir introduit le Quatuor de Schoenberg sur une scène d'une telle importance. Geissler ne trouvait aucune valeur artistique à Salomé, c'est pourquoi il en attribua le succès public à l'ambiance décadente 
du palais d'Hérode: "l'atmosphère doucereuse et redoutable " qui s'en dégageait provoquait une libération des "émotions et états d'âme dans lesquels des millions de gens ont aujourd'hui sombré ». L'absence de contrôle (« les véritables orgies (sic) ») dans la « cacophonie », pouvait-on lire dans son deuxième compte rendu du Quatuor, donnait l'impression que les quatre musiciens jouaient ad libitum - un terme associé à des pratiques "primitives", issues d'un lointain folklore, mais pas à l'art musical de tradition occidentale. «Il n'y avait pas d'oasis dans ce désert », et le public allemand - y compris la princesse de Saxe, qu'il avait vue soupirer à plusieurs reprises au cours de l'exécution, « avait soif » de la fin du Quatuor.

Enfin, les innovations dans la polyphonie dérangeaient, non pas seulement parce que des compositeurs juifs accomplis, prétendument trop intellectuels, s'appropriaient l'inestimable héritage musical allemand, mais parce que des ethnomusicologues commençaient à considérer la polyphonie comme une partie des musiques du monde. Le terme « hétérophonie » intégra le vocabulaire ethnomusicologique dès 1901, lorsque Carl Stumpf distingua les techniques utilisées par des musiciens du Siam (alors en visite à Berlin) de la «polyphonie pure ${ }^{35}$ ». Son usage s'étendit encore en 1908 avec Guido Adler, qui reprochait au contrepoint de la musique moderne son hétérophonie, c'est-àdire le fait qu'il procédât par empilement des voix au lieu de les placer les unes contre les autres. Ainsi les compositeurs eurent-ils recours à des "formes et moyens d'expression primitifs", à l'instar des sécessionnistes qui, dans les arts visuels, utilisaient des formes « exotiques, et plus spécifiquement asiatiques ${ }^{36} »$. Adler formula une théorie polémique selon laquelle les origines de la musique occidentale, autrement dit de la polyphonie, provenaient de l'hétérophonie de musiques folkloriques et extraoccidentales. Mais il finit par réaffirmer la valeur unique de l'art musical occidental, dont «le haut degré d'évolution» le plaçait bien au-dessus de l'hétérophonie des musiques primitives et folkloriques ${ }^{37}$. Étant donnée cette conscience accrue de la musique extra-occidentale, et ce au-delà du seul domaine de l'ethnomusicologie, démarquer le champ de la musique allemande devint un enjeu majeur pour l'hégémonie allemande, aussi bien dans le domaine de la musique qu'au point de vue politique. Le contrepoint requerrait structuration et anticipation, là où l'« hétérophonie " pouvait être improvisée. L'hétérophonie était comprise comme le procédé d'accompagnement d'un chant mélodique par son ornementation ${ }^{38}$. Elle ne répondait donc ni à la logique du contrepoint, ni à la nécessité d'une équivalence structurelle des voix. Le terme d'hétérophonie englobait également l'interaction étrange et indisciplinée des voix dans la musique de chambre de Schoenberg. En effet, le compositeur juif ne parvenait pas à écrire du contrepoint ; tout au plus pouvait-il créer des voix ornementales indépendantes, dans le style de la musique folklorique. Un critique évoquait, à propos de la création berlinoise de l'opus 7 , la façon dont Schoenberg "entrecroise l'absence de raffinement avec le plus grand raffinement » sans respecter l'esprit de la musique allemande : il est donc finalement « incapable de construire quoi que ce soit.» De plus, «il veut ériger en principe un procédé qu'il n'utilise qu'occasionnellement (l'hétérophonie dépourvue de raffinement).» Cela, poursuivait le critique, est contraire à l'art, car un "véritable artiste » ne devrait pas travailler sur prescription ${ }^{39}$.

Les hommages juifs rendus à Schoenberg n'ont fait que confirmer ces accusations. Les caractéristiques de l'écriture de Schoenberg, réputées typiques de l'Est, étaient significatives pour Heinrich Berl, comme en témoigne Das Judentum in der Musik, son réquisitoire sioniste contre l'antisémitisme de Wagner. « Mahler a dit un jour qu'il n'y a 
pas d'harmonie, « seulement » du contrepoint ; en vérité, Schoenberg est " seulement » un contrapuntiste, pas un harmoniste. » L'auteur soulignait avec emphase que la crise de la musique européenne se trouvait en grande partie résolue avec la polyphonie de Schoenberg. Dans le Quatuor à cordes $n^{\circ} 2$, la Symphonie de chambre $n^{\circ} 1$, ainsi que dans la Sérénade, Schoenberg s'impose en véritable contrapuntiste : «il remonte aux débuts de la musique, à ses origines orientales et primitives." Pour Berl, les origines de la polyphonie ne sont pas du côté de l'Occident ; la musique orientale est hétérophonique et correspond parfaitement à la polyphonie de Schoenberg ${ }^{40}$. Ces propos font écho aux affirmations du compositeur dans l'édition de 1922 de son Traité d'harmonie :

nous entrons dans une nouvelle ère du style polyphonique; comme auparavant, dans les temps anciens, les harmonies seront le produit de la voix principale : elles ne seront justifiées que par les lignes mélodiques ${ }^{41}$ !

Ce qui est unique dans la polyphonie de Schoenberg, selon Berl, c'est que « chaque voix et chaque instrument a sa propre signification, qu'ils développent librement de façon purement linéaire ${ }^{42}$ ». Un an plus tard, dans un article intitulé "De Wagner à Schoenberg", Weißmann affirmait que Schoenberg avait réfuté les assertions antisémites de Wagner : son «atonalité et sa linéarité » avaient une grande influence et, en général, son art était régi par des principes profondément éthiques ${ }^{43}$. Pour cette génération, qui cherchait maîtrise et puissance à partir du développement musical, la linéarité était l'élément crucial sous-tendu par l'écoute contrapuntique.

\section{Polyphonie et abstraction}

En tant qu'ensemble d'événements qui résistent à l'intégration et n'offrent ainsi aucune perspective unifiée, la nature de la polyphonie offre à l'abstraction un fondement conceptuel. Il n'est pas surprenant qu'écrivains et peintres se soient tournés vers d'autres formes d'art pour trouver des analogues à leurs propres innovations. Au début du vingtième siècle, l'abstraction n'est pas tant une qualité intrinsèque à l'œuvre qu'un processus, une orientation de l'artiste. Pour Mahler, qui baignait dans l'univers artistique et littéraire d'un romantisme tardif, le détachement se produisit à travers l'ironie et dans un médium purement instrumental. Dans le cas de Schoenberg, comme le signale Adolf Weißmann, le cheminement vers l'abstraction le conduisit à la musique de chambre et à des «combinaisons contrapuntiques. " C'est là un autre aspect du "Schoenberg romantique", poursuit Weißmann, «nourri de pure musique de chambre, indépendant de tout colorisme et voué à la théorie et à l'abstraction... Son approche de la forme manifeste une tendance au traitement horizontal; il cherche ainsi, quoiqu'avec une réserve moderne, à rejoindre Bach et le style épuré du dernier quatuor à cordes de Beethoven ${ }^{44}$. »

57 Le détachement vis-à-vis du matériau musical, en tant que mode de pensée abstraite, s'exprime souvent en termes spatiaux, du moins pour les musiciens. L'aspect sans aucun doute le plus innovant du style de Mahler, consistant à faire entendre simultanément des thèmes (ou des timbres, comme dans sa manière tardive) radicalement différents, relève de cette pensée de l'espace. C'était également le cas de Schoenberg, hormis pour le contrepoint, pour lequel il s'en remettait strictement à la tradition allemande de Bach. Schoenberg rejetait l'idée de linéarité, métaphore caractérisant au mieux l'écoute contemporaine du contrepoint, et insistait, contre la nature de sa propre écriture, sur le fait que le contrepoint devait être identifiable à 
l'oreille en terme de point contre point. Néanmoins, lorsqu'il développa sa théorie des douze tons, il eut recours aux métaphores spatiales pour suggérer un sentiment de liberté totale. L' «unité de l'espace musical» rendue possible par la composition à douze tons n'était pas sans évoquer le paradis de Swedenborg, tel que Balzac le désigne dans Séraphîta: «Il n'y a pas de fond ultime, ni de gauche ni de droite, ni d'avant ni d'arrière. » Puis :

pour la faculté imaginative et créatrice, ces relations dans la sphère matérielle sont aussi indépendantes des directions et des plans que peuvent l'être, pour nos facultés perceptives, les objets matériels dans leur propre sphère ${ }^{45}$.

Les critiques musicaux firent preuve d'une remarquable ouverture d'esprit envers à ce corrélat de l'abstraction dans les arts visuels, à savoir, une composition dont les règles et la logique interne demeuraient insaisissables. Le Premier Quatuor de Schoenberg exerçait tout de même un attrait pour ceux à qui sa structure résistait ; ils flétrissaient alors la musique sur le plan compositionnel. L'exécution d'une œuvre nouvelle leur plut immensément, mais la musique en elle-même les rebuta. Comment une telle distinction est-elle possible lors d'une création, alors que l'on ne connait pas encore l'œuvre (ou, au mieux, mais c'est peu probable, en ayant étudié sa partition)? Dans les comptesrendus vivants et sensibles de leur expérience d'auditeur, les détracteurs des symphonies de Mahler concédèrent la valeur de l'oeuvre exécutée, quand bien même ils lui déniaient tout droit de cité au répertoire. La raison d'un tel décalage entre la réaction au concert et la réaction à la musique elle-même est sans doute en partie liée à la fascination exercée par la puissance du chef, au contrôle de telles masses orchestrales. Quant à l'opus 7 de Schoenberg, curieusement, c'est son exécution qui plut, et non pas ce qui fut joué. Louis admit que ce qu'il avait entendu ne pouvait pas être rendu par des mots, mais c'est à l'interprétation qu'il en attribua le mérite : « Les mots sont impuissants à donner la moindre idée de la façon dont le quatuor viennois Rosé a interprété cette œuvre d'une difficulté sans précédent. Jamais encore je n'avais entendu jouer un quatuor de façon si incroyable. " Il écrivit ces lignes dans la même année qui vit l'achèvement de son livre Die Deutsche Musik der Gegenwart, un ouvrage violemment antisémite duquel Schoenberg était complètement exclu.

Si le contrepoint favorisait les considérations ethniques, ça n'était pas seulement le fait des tendances antisémites ou nationalistes des auditeurs. Il y avait dans le contrepoint un caractère abstrait engendré par la divergence radicale des voix. Dans une certaine mesure, l'auditeur est insignifiant pour ce type de musique, tant son écoute est incapable de rassembler les divers fils : l'écoute synthétique devient inopérante. Cette musique comporte de nouvelles dimensions, elle divise la conscience en deux parties, voire davantage. Autrement dit, il n'y a plus de point de repère à partir duquel les autres voix puissent être comprises. La polyphonie moderne, pour ainsi dire, contraint la perception à saisir au moins deux événements (ou processus) linéaires isolés mais conçus pour fonctionner ensemble - une écoute bien plus abstraite que dans la musique atonale, car après tout celle-ci peut être ancrée dans l'expression d'émotions et une approche sensorielle, à travers le timbre, les contours mélodiques ou le rythme.

Cette marche vers l'abstraction, vers une pureté de l'expression artistique, favorisa la tolérance à l'incompréhensibilité. Dans un texte de 1906, "Le poète contemporain ", Hugo von Hofmannstahl admire la science pour son "élan vers les mathématiques ", qu'il compare à celui des arts vers la pureté - un autre chemin vers la musique. Hofmannsthal avance que la science mathématique est la seule qui soit fondée sur un élément humain ${ }^{46}$, comme si toutes les autres formes de connaissance (Wissenschaften) 
s'étaient sclérosées. Dans les années suivantes, l'énergie et la complexité de la modernité musicale et artistique symbolisent souvent la liberté des individus d'explorer la musique par delà les règles et les concepts. Lors d'une exécution du Premier Quatuor à cordes de Schoenberg pendant la Première Guerre mondiale, un critique exprima sa nostalgie du temps où l'aspect "mathématique de la composition » de cette musique frappait les auditeurs, plutôt que " son calme et sa noblesse », comme c'était désormais le cas. C'est précisément parce qu'elle était incompréhensible que cette musique était si saisissante dix ans auparavant ${ }^{47}$.

61 L'attitude de Schoenberg quant au rôle de la compréhensibilité dans la perception artistique a profondément évolué au fil du temps. Dans une lettre de 1920 adressée à Berg à propos de son Premier Quatuor, il dénigre l'attitude d'écoute "moderne ", qui "s'enferme dans l'abscond et ne se réjouit qu'au prix de l'incompréhension des autres. " Schoenberg regrette l'éloge prétendument superficiel des effets de timbre et des sections lentes ${ }^{48}$. Son souci de voir son œuvre reconnue sur le plan structurel, plutôt qu'expressif, n'a donc rien de surprenant, car il s'était secrètement assigné un plan et avait renoncé au chemin pris jusque-là d'une musique à programme. C'est seulement plus tard qu'il s'éprit d'une telle incompréhensibilité, lors d'un tournant significatif de sa vie, et au moment d'un intérêt renouvelé pour la politique. Dans l'un de ses premiers cours donnés après son installation aux États-Unis en 1933 - un texte dont il avait le sentiment de devoir le remanier sans cesse - il explique : «Je suis très heureux de comprendre si peu Einstein et Kant : cela me permet de penser qu'il y a là quelque chose." Dans une troisième version, il invoque une comparaison avec les mathématiques au motif que, là comme en musique, ce qui est important doit être incompréhensible ${ }^{49}$. Durant ses premières années aux États-Unis, Schoenberg repensait avec nostalgie aux temps où sa musique était incomprise. C'est seulement plus tard, songeait-il, «lorsque les gens prétendirent comprendre les théories d'Einstein et la musique de Schoenberg, que j'ai connu la solitude ${ }^{50}$. »

62 De fait, c'est le caractère incompréhensible du contrepoint qui permit à la musique de s'ériger en modèle pour les autres arts, et ce, non pas de façon purement poétique, comme l'art immatériel au cœur de la pensée romantique, mais dans un sens plus spécifique et procédural. Le contrepoint servait en effet de métaphore pour l'abstraction dans les arts et la littérature. L'inspiration venait moins de l'idée de la musique que de l'expérience d'écoute. La Cinquième Symphonie de Mahler poussa Kalbeck à se demander si son orchestration était plus semblable à du Klimt ou à du Klinger. Cependant, sa Sixième Symphonie conduisit Korngold à rapprocher le contrepoint du style de Klimt :

Il y a à Vienne un peintre comparable à Mahler par la confusion contrapuntique des lignes instrumentales et par son défi aux Philistins : Klimt. Mais Klimt n'en a ni le pathos, ni l'énergie directrice, ni le douloureux agacement des nerfs ${ }^{51}$.

63 La complexité seule n'était pas l'accès au modernisme, mais une simultanéité qui s'illustre à travers l'écoute contrapuntique. Dans le premier texte publié de James Joyce, Chamber Music (1907), écrit pour être mis en musique, le style, d'abord simplement lyrique, évolue progressivement vers la complexité. Mais dix ans plus tard, dans Ulysse, l'auteur recourt à des techniques de contrepoint et de fugue pour dresser un portrait radical du temps et de l'espace qui changera le cours de la prose moderne. Dans la polyphonie moderne, l'auditeur est constamment repositionné, sans orientation spatiale claire de la basse ou de la mélodie. La gêne, ou du moins la sensation physique qu'implique l'écoute d'une telle ligne de basse est brillamment 
rendue dans un fragment de Arnold Zweig de 1913 et consacré à l'opus 7 de Schoenberg. L'unique changement de narration, qui passe alors de la troisième à la première personne, intervient au moment où le protagoniste éprouve la nécessité de se mouvoir pour réagir à l'élan et à la puissance du violoncelle :

Le violoncelle se courbe comme une vague tel l'arc des ponts qui traversent toutes les rivières, le pied monte sur leurs structures d'acier et le train vrombit d'un lit de rivière à l'autre, plein de marchandises et de voyageurs montés à bord - j'y posai moi-même bientôt le pied ${ }^{52}$.

Pour Zweig, le contrepoint renvoie également à l'idée d'une navigation du moi dans et entre les lignes ainsi qu'une conception évolutive de leur ensemble. L'écoute contrapuntique implique-t-elle une écoute corporelle? On se souvient de la représentation $\mathrm{du}$ contrepoint dans un croquis de Schoenberg: une figure contorsionnée jouant du piano ${ }^{53}$.

Le contrepoint était d'une certaine façon un meilleur corrélat pour l'abstraction dans les arts visuels que le cliché de l'atonalité. Kandinsky et Klimt eurent tous deux recours à ce procédé en tant que métaphore pour leurs premières incursions dans le domaine de l'abstrait ${ }^{54}$. Kandinsky reçut d'une expérience de l'opus 7 au concert l'impulsion à l'origine de sa longue correspondance avec Schoenberg et il semble clair que l'inspiration lui vint particulièrement de son écriture contrapuntique :

Dans vos œuvres, vous avez réalisé ce que depuis si longtemps j'appelle de mes vœux en matière de musique, quoique sans pouvoir vraiment le définir. La vie indépendante des voies dans vos compositions, leur développement indépendant à travers leur propre devenir, voila exactement ce que je recherche dans ma peinture $^{55}$.

L'indépendance des voies signifiait l'incapacité de synthétiser la musique en un seul plan, donc une certaine forme de relativité. Il ne serait pas surprenant de voir des critiques musicaux de la presse généraliste allemande et autrichienne faire un sort aux implications des développements récents de la physique, à l'origine d'un renouvellement fondamental de la façon de penser l'univers. En outre, d'autres avaient déjà anticipé la relativité du temps et de l'espace sous des formes plus évocatrices pour la perception artistique et la création. Ernst Mach, le directeur de thèse de David Josef Bach, à l'époque où ce dernier était très ami avec Schoenberg, a démontré que les espaces auditifs, visuels et tactiles varient dans leur temps de réaction. La publication de ces idées dans l'ouvrage Space and Geometry in Light of Physiological, Psychological, and Physical Inquiry (1901), proposait effectivement un fondement physiologique à l'espace


temps et de l'espace laissait place à des échelles relatives, en fonction de la position de leur observateur, suggérait que l'auditeur devait renoncer à l'idée d'une méthode unique d'écoute, tout comme semblait l'indiquer le nouveau contrepoint ${ }^{57}$.

L'idée de contrepoint fascinait également Paul Klee, violoniste amateur, dans son exploration entre figuration et abstraction. Dans Das bildnerische Denken, il définit le " polyphonique » comme « la pénétration par plusieurs voix de différentes nuances de couleurs, doté d'un point centrifuge et d'une lumière transparente. » La polyphonie était une façon de créer du mouvement ou, en termes plus abstraits, une directionnalité, comme en témoigne son Polyphon-Abstractes de 1930. Klee le soulignait lui-même, il aspirait à bien plus qu'à la simple la transposition de la polyphonie dans le domaine du visuel. Plus exactement, la conscience de la polyphonie devait aider l'artiste à « pénétrer profondément dans la sphère cosmique de façon à y introduire un 
contemplateur de l'art transformé. Le principe du contrepoint ou de la polyphonie est présent partout, pas seulement dans la musique. $\aleph^{58}$ Le contrepoint moderne facilitait le besoin de saisir l'espace, le temps, la vision et le son dans un nouveau programme rationaliste et sensoriel. Dans le domaine musical, à la fois précurseur et modèle, les innovations liées au contrepoint entre 1905 et 1907 ont joué un rôle spécifique dans la reformulation conceptuelle de l'écoute. Car, malgré le contexte idéologiquement chargé dans lequel il s'inscrivait, le contrepoint avait le potentiel de reconfigurer l'orientation spatiale, dévoilant ainsi la relativité de sa véritable nature et, par ses ruptures et ses lignes heurtées, insistait sur ce point que l'individu ne saurait être absorbé dans le tout.

\section{ANNEXES}

\section{Annexe 1 - Comptes rendus cités}

\section{Mahler ${ }^{59}$}

Allgemeine Musik-Zeitung 34, nº 4, 25 jan. 1907, p. 62 [-rm-]. Sixième Symphonie

ALTMANN, Gustav, Die Musik, Vol. 4, n 15, 1905, p. 45 [Cinquième Symphonie] ; vol. 5, $\mathrm{n}^{\circ}$ 19 (1ère parution de juin 1906), p. 49-50 [Sixième Symphonie].

BACH, David Josef, Arbeiter Zeitung, 10 jan. 1907. Sixième Symphonie.

BATKA, Richard, Prager Tageblatt, 20 sept. 1908, Septième Symphonie.

BRANDES, Friedrich, Der Kunstwart (2e parution de juillet 1906). Sixième Symphonie.

HELM, Theodor, Musikalisches Wochenblatt 37, n² 2, 11 jan. 1906, p. 34. Cinquième Symphonie.

HIRSCHFELD, Robert, Wiener Abendpost, 20 nov., 1900, Première Symphonie.

KALBECK, Max, Neues Wiener Tagblatt, 7 jan. 1907, Sixième Symphonie.

KORNGOLD, Julius, Neue Freie Presse, 12 déc. 1905 [Cinquième Symphonie], 8 jan. 1907

[Sixième Symphonie], 6 nov. 1909 [Septième Symphonie], 16 déc. 1930 [Septième Symphonie].

LIEBSTOCKL, Hans, Illustriertes Wiener Extrablatt, 5 jan. 1907. Sixième Symphonie.

LOEWENGARD, Max, Der Hamburgische Correspondent, 13 mars 1905, Cinquième Symphonie. MUNTZ, Maximilian, Deutsche Zeitung, 14 déc. 1905, Cinquième Symphonie.

VANCSA, Max, Die Wage 10, n² 2, 1907, p. 37-38. Sixième Symphonie. 


\section{Schoenberg et Strauss ${ }^{60}$}

Berliner Borsen-Courier, 3 juillet 1907 [F.Z.]. Une version révisée fut rééditée dans Breslauer Morgen Zeitung, 4 juillet 1907.

BRANDES, Friedrich, Deutsche Tageszeitung, 6, juillet 1907 ; réédité dans Signale fur die Musikalische Welt 65, n 46/47, 24 juillet 1907.

Dresdner Nachrichten, 1er juillet 1907 [H. St.].

GEISSLER, F. A., Leipziger Tagblatt, 1er juillet 1907.

Hamburger Nachrichten, 2 juillet 1907.

HIRSCHFELD, Robert, compte rendu de Salome de Strauss, in Wiener Abendpost, 26 mai 1907 ; trad. dans BOTSTEIN, Leon, « Richard Strauss and the Viennese Critics (1896-1924) ", in Richard Strauss and His World, ed. Bryan Gilliam, Princeton, Princeton University Press, 1992, p. 333-336.

KAUDERS, Albert, Fremden-Blatt, 11 fév. 1907.

KORNGOLD, Julius, «Richard Strauss's Salome : A Conversation », in Neues Wiener Tagblatt, 28 mai 1907 ; trad. dans BOTSTEIN, « Strauss and the Viennese Critics », p. 342-349.

Der Kunstwart 20, $\mathrm{n}^{\circ}$ 13, 1er avril 1907, « Neuheiten des Wiener Musikjahres ».

LOUIS, Rudolf, Münchner Neueste Nachrichten, 6 juillet 1907.

MARSCHALK, Max, Vossische Zeitung, 22 fév. 1907,

Montags Revue, 11 fév. 1907 [H.v.F.-A.].

Neue Musik-Zeitung, 1907 [O.K.].

PÜRINGER, August, Allgemeine Musik-Zeitung, 22 mars 1907.

RIEZLER, Walter, « Von neuer deutscher Musik », Halbmonatschrift für deutsche Kultur, Mars, no. 16.

TISCHLER, Gerhard, Rheinische Musik-und Theater-Zeitung, p. 357-359.

Das Vaterland, 12 fév. 1907.

WEIßMANN, Adolf, « Aus dem Berliner Musikleben », coupure non identifiée, sur la création berlinoise de l'opus 7 de Schoenberg.

Wiener Allgemeine Zeitung, 23 fév. 1907 [C.L.].

\section{Annexe 2 - Tableau chronologique des concerts}

\begin{tabular}{|l|l|l|}
\hline Saison 1905-1906 & \multicolumn{2}{|l|}{} \\
\hline Strauss : Salomé & création (Dresde) & \\
\hline Mahler : Sixième Symphonie & création (Dresde) & Festival des compositeurs du ADM ${ }^{61}$ \\
\hline Saison 1906-1907 & \multicolumn{2}{|l}{} \\
\hline
\end{tabular}




\begin{tabular}{|l|l|l|}
\hline Mahler : Sixième Symphonie & Création viennoise & \\
\hline Schoenberg : opus 7 & Création (Vienne) & \\
\hline Schoenberg : opus 9 & Création (Vienne) & \\
\hline Strauss : Salomé & Création viennoise & \\
\hline Strauss : Salomé & Dresde & Festival des compositeurs du ADM \\
\hline Schoenberg: opus 7 & Création allemande (Dresde) & Festival des compositeurs du ADM \\
\hline
\end{tabular}

ADM : ALLgEMEINE DEUTSCHE MUSIKVEREIN

\section{NOTES}

1. BRINKMANN, Reinhold, « Ein “Letzer Riese in der Musik”. Gedanken zu Max Reger im 20. Jahrhundert ", in WAGNER, Günther, (éd.), Jahrbuch des Staatlichen Instituts für Musikforschung Preußischer Kulturbesitz, Stuttgart et Weimar, Verlag J.B. Metzler, 2000, p. 18.

2. KILLIAN, Herbert (éd.), Gustav Mahler in den Erinnerungen von Natalie Bauer-Lechner, Hamburg, K.D. Wagner, 1984, p. 189. Cette traduction et les suivantes sont tirées de l'ouvrage de BAUER-LECHNER, Natalie, Recollections of Gustav Mahler, éd. Peter Franklin, trad. Dika Newlin, London, Faber Music, 1980.

3. SCHOENBERG, Arnold, « National Music » 2 (24 février 1931), in STEIN, Leonard, Style and Idea. Selected Writings of Arnold Schoenberg, trad. Leo Black, Los Angeles et Berkeley, Univeristy of California Press, 1984, p. 173.

4. MAHLER, Alma, Gustav Mahler : Erinnerungen und Briefe, Amsterdam, Allert de Lange, 1949 , p. 243.

5. Mahler in den Erinnerungen von Bauer-Lechner (voir plus haut, note 3).

6. Henry et Sydney Cowell, qui rapportent cette expérience du père d'Ives, voient dans cet épisode d'enfance l'origine de l'idée complexe que se fait le compositeur de la polyphonie. Voir leur ouvrage Charles Ives and His Music, New York, Oxford University Press, 1969, p. 144-145. Mahler in den Erinnerungen von Bauer-Lechner (voir plus haut, note 3), p. 165.

7. DRAESEKE, Felix, Der gebundene Styl. Lehrbuch für Kontrapunkt und Fuge, vol. 1, Hanovre, Louis Oertel, 1902, p. 5.

8. GRUNSKY, Karl, Musikästhetik, Leipzig, G.J. Göschen, 1907, p. 75-76. L'ouvrage fut imprimé quatre fois, d'abord en 1907, puis en 1909 et 1911, et en 1923 dans une édition révisée.

9. NORDAU, Max, Entartung, 2e éd., Berlin, C. Duncker, 1893, p. 24-25.

10. DRAESEKE, Der gebundene Styl (voir plus haut, note 8), p. 5-6.

11. GRUNSKY, Musikästhetik (voir plus haut, note 9), p. 71-72, 77, 74.

12. WEIßMANN, Adolf, Problems of modern music, trad. M. Bozman, Londres, Toronto et New York, 1925, p. 83-84. 
13. LOUIS, Rudolf, Anton Bruckner, Munich et Leipzig, Georg Müller,1905, p. 196-197.

14. STEPHAN, Rudolf, "Schoenbergs Entwurf über "Das Komponieren mit selbständigen Stimmen" ", Archiv für Musikwissenschaft, $\mathrm{n}^{\circ}$ 29, 1972, p. 239.

15. Les principaux textes utilisés dans les conservatoires allemands étaient BELLERMAN, Heinrich, Der Kontrapunkt, oder Anleitung zur Stimmführung in der musikalischen Composition, Berlin, Verlag von Julius Springer, 1862, réimprimé en 1902, et RIEMANN, Hugo, Große Kompositionslehre, vol. 2, Der polyphone Satz (Kontrapunkt, Fuge und Kanon), Berlin et Stuttgart, W. Spemann, 1903.

16. Les associations liées au contrepoint, ou peut-être l'idée selon laquelle la musique de Mahler représentait une menace, passèrent de mode, et Korngold révisa sa formule dans les années 1930 pour y voir « une individualisation et une opposition sans limite des voix orchestrales ».

17. Voir la lettre de Schoenberg à Pierre Ferroud, 31 août 1922, in STEIN, Erwin (éd.), Letters, trad. Eithne Wilkins et Ernst Kaiser, Londres, Faber and Faber, 1964, p. 76-77.

18. BRINKMAN, Reinhold, "The compressed Symphony: On the historical Content of Schoenberg's Op. 9 », trad. Irene Zedlacher, in FRISCH, Walter (éd.), Schoenberg and His World, Princeton, Princeton University Press, 1999, p. 142-143.

19. «Der Fall Schoenberg », coupure non identifiée, Centre Arnold Schoenberg, Vienne.

20. La personnification des deux thèmes à une jeune fille violée et à un mâle exploité est renforcée par le choix des mots : das junfräuliche Thema, le pronom neutre faisant allusion à das Mädchen et à der Gedanke. L'auteur identifie le point culminant, « rendue folle ", à un jeu de mots (verrückt, littéralement, être déplacé).

21. VOGLER, Abbé, System für den Fugenbau, Offenbach-sur-le-Main, ca., 1817, p. 28.

22. Un tel contrepoint était l'une des formes du manque de rigueur et de substance de la nouvelle musique: recours aux pédales au lieu de voix de basse élaborées et d'harmonies changeantes; aux ostinatos, séquences, et fugatos au lieu de variations développées ; et à la dissonance pour masquer la banalité du matériau thématique. Voir SCHOENBERG, « New Music, Outmoded Music, Style and Idea », 1946, in STEIN, Style and Idea (voir plus haut, note 4), p. 120. Cet ouvrage est une version revue et corrigée d'un cours donné à Prague et à Vienne en 1933, ainsi qu'à Boston en 1933 ou 1934.

23. WAGNER, Richard, Richard Wagner's Prose Works, vol. 3, The Theatre, Londres, Kegan Paul, Trench, Trübner \& Co., 1894, p. 88-89, trad. William Ashton Ellis.

24. Strauss aurait fait ce commentaire en 1939, lors d'une conversation avec Hans Severus Ziegler, qui organisa l'exposition. Voir la lettre du 18 janvier 1965 de Ziegler à Fred K. Prieberg, commentée dans PRIEBERG, Musik im NS-Staat, Francfort-sur-le-Main, Fischer Taschenbuch Verlag, 1982, p. 212.

25. Conversation personnelle avec le Professeur Gilliam : GILMAN, Sander L., "Strauss and the Pervert ", in GROOS, Arthur et PARKER, Roger (éd.), Reading Opera, Princeton, Princeton University Press, 1988, p. 319-320.

26. Sur Hirschfeld, voir BOTSTEIN, Leon, Music and Its Public: Habits of Listening and the Crisis of Musical Modernism in Vienna, 1870-1914, thèse de doctorat, Université d'Harvard, 1985.

27. Voir la lettre du 9 novembre 1829 de Goethe à Zelter.

28. LOUIS, Rudolf, Die Deutsche Musik der Gegenwart, Munich et Leipzig, Georg MüllerVerlag 1909, p. 182. 
29. SCHEICHL, Sigurd Paul, «Contexts and Nuances of Anti-Jewish Language : Were all the 'Antisemites' Antisemites?", in BOTZ, Gerard, OXAL, Viar et POLLACK, Michael (éd.), Jews, Antisemitism and Culture in Vienna, Londres et New York, Routledge \& Kegan Paul, 1987, p. 94.

30. Hitler et Pfitzner ont commenté Weininger en février 1923. Pfitzner a dédicacé une copie de Huttens letze Tage à "Adolf Hitler, le grand Allemand", qu'il désirait lui envoyer pendant son emprisonnement. C'est n'est qu'après la chute du Troisième Reich que Pfitzner annonce son mépris pour l'incapacité de Hitler à comprendre la nature abstraite de l'antisémitisme de Weininger. WILLIAMSON, John, The Music of Hans Pfitzner, Oxford, Clarendon Press, 1922, p. 325.

31. MAYER, Sigmund, Die Wiener Juden, Kommerz, Kultur, Politik, 1700-1900, Vienne et Berlin, R. Löwit, 1917, p. 475.

32. Ces modes expérimentaux sont décrits de façon explicite dans les déclarations que Püringer attribue à Schoenberg: "Qu'y puis-je, au fait que le public ne perçoive pas encore mon dessein thématique, qu'il ne pénètre pas ma polyphonie, qu'il n'entende pas mon rythme, qu'il ne saisisse pas fermement ma tonalité ?»

33. L'anecdote est rapportée par Richard Batka, qui y voit la preuve que Mahler, bien que bohémien, était accepté comme un « représentant de l'art moderne allemand ». La création de la Septième Symphonie à Prague revêt donc pour Batka les traits d'un « événement politique ».

34. Il semble en tous cas que les raisons de santé invoquées par Strauss dans un télégramme n'aient pas été satisfaisantes aux yeux du critique de droite Gerhard Tischer, qui écrivit dans son compte rendu : «Richard le Second [...] aurait aussi pu présider au festival s'il n'avait pas plaidé, comme il arrive fréquemment, en fin de règne, l'épuisement lié à son métier et le fardeau de sa charge en guise d'excuses. » En fait, Strauss a dû se sentir extrêmement préoccupé d'avoir négligé son travail sur Elektra alors qu'il faisait face au succès de Salomé. Ses carnets d'esquissent montrent qu'il travaillait à la scène de Clytemnestre en juin 1907. Je suis reconnaissante à Alex Ross pour ses remarques sur ce point.

35. Stumpf commet une erreur dans sa lecture de Platon en conférant une autorité classique à une idée moderne. STUMPF, Carl, « Tonsystem und Musik der Siamesen », in Beiträge zur Akustik und zur Musikwissenschaft, vol. 3, Leipzig, 1901, p. 131; rpt. dans STUMPF, Carl et MORITZ, Erich (éd.), Sammelbände fur Vergleichende Musikwissenschaft, vol. 1, Munich, 1922, p. 22-77. Comme l'explique Frieder Zaminer, le passage de Platon, fréquemment cité, ne permet pas une interprétation claire du terme hétérophonie. Quoi qu'il en soit, le mot n'avait assurément dans l'Antiquité aucune connotation musicale. Voir ZAMINER, « Heterophonie », in RIEMANN, Musiklexikon, Sachteil, éd. Hans Heinrich Eggebrecht, 12e éd., Mainz, 1967, p. 371.

36. ADLER, Guido, "Über Heterophonie », Jahrbuch der Musikbibliothek, Peters, 1908, p. 17-18. Sur les Sécessionnistes dans la musique, voir également la critique de l'opus 7 de Schoenberg dans Der Kunstwart.

37. ADLER, « Über Heterophonie » (voir plus haut), p. 24.

38. RIEMANN, Hugo, Musik-Lexikon, 6e éd., Leipzig, 1905, « Heterophonie », p. 565.

39. Coupure de presse non identifiée, A. Sn, Centre Arnold Schoenberg.

40. BERL, Heinrich, Das Judentum in der Musik, Stuttgart, Deutsche Verlags-Anstalt, 1926, p. 171. 
41. SCHOENBERG, Arnold, Harmonielehre, 3e éd., Vienne, Universal Edition, 1922, p. 466.

42. BERL, Heinrich, Das Judentum in der Musik, Stuttgart, Deutsche Verlags-Anstalt, 1926, p. 171.

43. WEIßMANN, Adolf, "Von Wagner bis Schoenberg ", in Chemnitzer Volks-Zeitung, 5 Aug. 1927 (Steininger Sammlung, Preussische Geheimes Staatsarchiv).

44. WEIßMANN, Problems of Modern Music (voir plus haut, note 13), p. 180-181.

45. SCHOENBERG, Arnold, «Composition with Twelve Tones (1) », 1941, repris in Style and Idea (voir plus haut, note 4), p. 223.

46. HOFMANNSTAHL, Hugo von, "Der Dichter und diese Zeit", 1906, repris in Gesammelte Werke. Prosa, vol. 2, Francfort, S. Fischer Verlag, 1951, p. 259.

47. Critique de l'Opus 7, auteur inconnu, 6 mai 1917, conservée au Steininger Sammlung.

48. Lettre à Berg du 6 décembre 1920, trad. Christopher Hailey, « Between Instinct and Reflection: Berg and the Viennese Dichotomy », in JARMAN, Douglas (éd.), The Berg Companion, Boston, Northeastern Univ Press, 1990, p. 227.

49. SCHOENBERG, Arnold, "Neue und veraltete Musik, oder Stil und Gedanke ", 10 février 1933, in Gesammelte Schriften, vol. 1, Stil und Gedanke. Aufsätze zur Musik, VOJTĚCH, Ivan (éd.), Francfort-sur-le-Main, S. Fischer, 1976, p.477. D'autres versions dactylographiées sont conservées au Centre Arnold Schoenberg.

50. SCHOENBERG, Arnold, "How one becomes lonely ", 11 octobre 1937, repris in Style and Idea (voir plus haut, note 3), p. 51. Voir également RIETHMÜLLER, Albrecht, «Hermetik, Schock, Faßlichkeit. Zum Verhältnis von Musikwert und Publikum in der ersten Hälfte des 20. Jahrhunderts ", Archiv für Musik-wissenschaft 37, 1980, p. 32-60.

51. Die Musik 6, $\mathrm{n}^{\circ}$ 11, 1er mars 1907, p. 327.

52. ZWEIG, Arnold, "Quattersatz von Schoenberg », in Novellen, vol. 1, Berlin, 1961, p. 336. Zweig ne spécifie pas le numéro de l'opus, mais son titre correspond à la référence de Schoenberg dans les premiers programmes du «Quartet in einem Satz »; de plus, il n'y a pas de référence à une quelconque composante vocale dans le récit de Zweig. Pour une clairvoyante analyse de cet écrit, voir BOTSTEIN, Leon, "Schoenberg and the Audience : Modernism, Music and Politics in the Twentieth Century ", in Schoenberg and His World, op. cit., p. 19-54.

53. Un croquis de Schoenberg de 1921 est reproduit dans le texte de NEFF, Severine, "Schoenberg as Theorist: Three Forms of Presentation ", in Schoenberg and His World, op. cit., p. 73.

54. Dans ce contexte, il est intéressant de mentionner l'observation de Helmuth Plessner, selon laquelle le moment décisif de l'histoire de l'art du vingtième siècle, qui s'observe de façon analogue chez Schoenberg et chez Joyce, consiste dans le développement de nouveaux moyens de perception qui remplacent ceux qui les ont précédés.

55. Kandinsky assista à la création munichoise de l'opus 7 de Schoenberg, interprété par le Quatuor Rosé, le 2 janvier 1911. Deux semaines plus tard, il commençait sa correspondance avec le compositeur. Voir SCHOENBERG, Arnold et KANDINSKY, Wassily, Briefe, Bilder und Dokumente einer außergewöhnlichen Begegnung, éd. Jelena HahlKoch, Salzburg et Vienne, 1980, p. 19-20. 
56. KERN, Stephen, The Culture of Time and Space, 1880-1918, Cambridge, Harvard University Press, 1983, p. 133-134.

57. Voir mon article intitulé «Form, Innovation, Modernism: Early responses to Schoenberg's First String Quartet ", in BRINKMANN, Reinhold et WOLFF, Christoph (éd.), Music of My Future: The Schoenberg Quartet and Trio, Cambridge, Harvard University Press, 2001, p . 25-38.

58. KLEE, Paul, Das Bildnerische Denken, éd. Jürg Spiller, Basel et Stuttgart, 1964, p. 296 et 374.

59. Ces journaux se trouvent au Vondenhoff Nachla $\beta$, dans la section musique de la Bibliothèque Nationale d'Autriche.

60. Sauf mention contraire, toutes les références qui suivent sont trouvables au Centre Arnold Schoenberg.

61. Allgemeine Deutsche Musikverein.

\section{RÉSUMÉS}

Au début du vingtième siècle, la polyphonie moderne - qui menace à la fois la tradition et la compréhensibilité - devient emblématique des problèmes engendrés par la nouvelle musique. Cet article examine l'idéologie sous-jacente à l'écoute du contrepoint en examinant particulièrement la réception de la saison de concerts 1906-1907 - et, en particulier, la façon dont la rhétorique des comptes rendus des créations de la Sixième Symphonie de Mahler et de Salomé de Strauss influença la réception du Premier Quatuor à cordes de Schoenberg. L'impression produite par Salomé (les débats sur l'antisémitisme et sur l'identité juive allemande que suscita l'œuvre) fut si forte qu'elle s'imposa aux critiques viennois et allemands dans leur façon de percevoir le contrepoint, et ce malgré la diversité de leurs points de vue. Plus largement, l'article aborde également les formes que prend l'antisémitisme au début du vingtième siècle, ainsi que la façon dont le contrepoint a influencé les courants musicaux d'avant-garde.

In the early twentieth century, modern polyphony became a symbol for the problems of new music, threatening both tradition and comprehensibility. This article examines the ideology behind listening to counterpoint, with a focus on reception sources from the concert season 1906-07-in particular, how the rhetoric from the premieres of Mahler's Sixth and Strauss's Salome influenced the reception of Schoenberg's First String Quartet. The influence of Salome (the antisemitism it spawned and the debate over Jewish-German identity) was so strong as to override the different attitudes towards counterpoint on the part of Viennese and German critics. Broader issues the article addresses are forms of antisemitism in the early twentieth century and how influenced the counterpoint artistic avant-garde outside music.

\section{INDEX}

Keywords : counterpoint, Mahler, polyphony, Schoenberg

Mots-clés : contrepoint, Mahler, polyphonie, Schoenberg 


\section{AUTEURS}

\section{KAREN PAINTER}

Professeure de musicologie à l'University of Minnesota, Karen Painter est diplômée de Yale University (1987) et Columbia University (1996). Elle a précédemment enseigné à Dartmouth College et Harvard University, assurant aussi la fonction de directeure de l'office of Research and Analysis for the National Endowment for the Arts. Auteure de Simphonic Aspirations: German Music and Politics, 1900-1945 (Harvard University Press, 2007), elle a travaillé sur les rapports entre musique, écoute et idéologie dans le contexte de l'histoire sociale allemande du dix-neuvième siècle, les débats culturels de la fin-de-siècle, la Première Guerre Mondiale, le socialisme austroallemand et le nazisme. Ses recherches incluent des compositeurs comme Mozart, Schubert, Wagner, Bruckner, Mahler, Schoenberg, Richard Strauss, Hindemith et Orff.

Parallèlement à son travail universitaire Painter a atteint un public mélomane plus large avec l'édition des volumes Mahler and His World (Princeton University press, 2002) et Late Thoughts: Reflections on Artists and Composers at Work (Getty Publications, 2004, co-édité avec Thomas Crow) et le colloque organisé pour le Ojai Music Festival et avec le Boston Symphony Orchestra. Painter a aussi donné des conférences avant-concert au Lincoln Center, le festival de Salzburg, Los Angeles Philharmonic, et Orange Country Performing Arts Center. 